\title{
A Perturbative Analysis of Modulated Amplitude Waves in Bose-Einstein Condensates
}

\author{
Mason A. Porter \\ mason@math.gatech.edu \\ School of Mathematics and Center for Nonlinear Science \\ Georgia Institute of Technology, Atlanta, Georgia 30332 \\ Predrag Cvitanović \\ School of Physics and Center for Nonlinear Science \\ Georgia Institute of Technology, Atlanta, Georgia 30332
}

June 12, 2018

\begin{abstract}
We apply Lindstedt's method and multiple scale perturbation theory to analyze spatio-temporal structures in nonlinear Schrödinger equations and thereby study the dynamics of quasi-one-dimensional BoseEinstein condensates with mean-field interactions. We determine the dependence of the amplitude of modulated amplitude waves on their wave number. We also explore the band structure of Bose-Einstein condensates in detail using Hamiltonian perturbation theory and supporting numerical simulations.
\end{abstract}

PACS: 05.45.-a, 03.75.Lm,05.30.Jp, 05.45.Ac

Keywords: nonlinear dynamics, Bose-Einstein condensates, chaos

Bose-Einstein condensates (BECs) were observed experimentally in 1995 using dilute vapors of sodium and rubidium. The macroscopic behavior of BECs at zero temperature is modeled by the nonlinear Schrödinger equation in the presence of an external potential. This model has proven to be an excellent one for most experiments on BECs. When the external potential is spatially periodic (e.g., due to an optical lattice, which may be created using counter-propagating laser beams), the spectrum of the BEC exhibits a band stucture (spatial resonance structure). This paper utilizes Hamiltonian perturbation theory and supporting numerical simulations to study this structure in detail.

\section{Introduction}

At low temperatures, particles in a dilute gas can reside in the same quantum (ground) state, forming a Bose-Einstein condensate. ${ }^{15,23,33,43}$ This was first observed experimentally in 1995 with vapors of rubidium and sodium. ${ }^{4,24}$ In these experiments, atoms were confined in magnetic traps, evaporatively cooled to tempuratures on the order of fractions of microkelvins, left to expand by switching off the confining trap, and subsequently imaged with optical methods. ${ }^{23}$ A sharp peak in the velocity distribution was observed below a critical temperature, incidating that Bose-Einstein condensation had occurred.

BECs are inhomogeneous, so condensation can be observed in both momentum and coordinate space. The number of condensed atoms $N$ ranges from several thousand to several million. Confining traps are usually approximated well by harmonic potentials. There are two characteristic length scales: the harmonic oscillator length $a_{h o}=\sqrt{\hbar /\left(m \omega_{h o}\right)}$ [which is on the order of a few microns], where $\omega_{h o}=\left(\omega_{x} \omega_{y} \omega_{z}\right)^{1 / 3}$ is the geometric mean of the trapping frequencies, and the mean healing length $\chi=$ $1 / \sqrt{8 \pi|a|} \bar{n}$, where $\bar{n}$ is the mean density and $a$, the (two-body) $s$-wave scattering length, is determined by the atomic species of the condensate. ${ }^{6,23,34,43} \mathrm{In}$ - 
teractions between atoms are repulsive when $a>0$ and attractive when $a<0$. For a dilute ideal gas, $a \approx 0$. The length scales in BECs should be contrasted with those in systems like superfluid helium, in which the effects of inhomogeneity occur on a microscopic scale fixed by the interatomic distance. ${ }^{23}$

If considering only two-body, mean-field interactions, a dilute Bose-Einstein gas can be modeled using a cubic nonlinear Schrödinger equation (NLS) with an external potential, which is also known as the Gross-Pitaevskii (GP) equation. BECs are modeled in the quasi-one-dimensional (quasi-1D) regime when the transverse dimensions of the condensate are on the order of its healing length and its longitudinal dimension is much larger than its transverse ones. ${ }^{9-11,23}$ In the quasi-1D regime, one employs the $1 \mathrm{D}$ limit of a 3D mean-field theory rather than a true 1D mean-field theory, which would be appropriate were the tranverse dimension on the order of the atomic interaction length or the atomic size. ${ }^{7,9-11,48}$

When examining only two-body interactions, the condensate wavefunction ("order parameter") $\psi(x, t)$ satisfies a cubic NLS,

$$
i \hbar \psi_{t}=-\left[\hbar^{2} /(2 m)\right] \psi_{x x}+g|\psi|^{2} \psi+V(x) \psi,
$$

where $|\psi|^{2}$ is the number density, $V(x)$ is an external potential, $g=\left[4 \pi \hbar^{2} a / m\right]\left[1+\mathcal{O}\left(\zeta^{2}\right)\right]$, and $\zeta=\sqrt{|\psi|^{2}|a|^{3}}$ is the dilute gas parameter. ${ }^{6,23,34}$ Because the scattering length $a$ can be adjusted using a magnetic field in the vicinity of a Feshbach resonance, ${ }^{27}$ the contribution of the nonlinearity in (1) is tunable.

Potentials $V(x)$ of interest in the context of BECs include harmonic traps, periodic potentials ("standing light waves"), and periodically perturbed harmonic traps. The existence of quasi-1D cylindrical ("cigar-shaped") BECs motivate the study of periodic potentials without a confining trap along the dimension of the periodic lattice. ${ }^{37}$ Experimentalists use a weak harmonic trap on top of the periodic lattice to prevent the particles from spilling out. To achieve condensation, the periodic lattice is typically turned on after the trap. If one wishes to include the trap in theoretical analyses, $V(x)$ is modeled by

$$
V(x)=V_{0} \sin \left(\kappa\left(x-x_{0}\right)\right)+V_{1} x^{2},
$$

where $\kappa$ is the lattice wave number, $V_{0}$ is the height of the periodic lattice, and $x_{0}$ is the offset of the periodic potential. (Note that these three quantities can all be tuned experimentally.) The periodic term dominates for small $x$, but the harmonic trap otherwise becomes quickly dominant. When $V_{1} \ll V_{0}$, the potential is dominated by its periodic contribution for many (20 or more) periods. ${ }^{14,18,25}$ (For example, when $V_{0} / V_{1}=500, \kappa=10$, and $x_{0}=0$, the harmonic component of $V(x)$ essentially does not contribute for 10 periods.) In this work, we usually let $V_{1}=0$ and focus on periodic potentials. Spatially periodic potentials have been employed in experimental studies of $\mathrm{BECs}^{3,32}$ and have also been studied theoretically. $^{2,8-11,18,20,25,40,49}$

When the optical lattice has deep wells (large $\left|V_{0}\right|$ ), an analytical description of BECs in terms of Wannier wave functions can be obtained in the tight-binding approximation. ${ }^{41}$ The Bose-Hubbard Hamiltonian, which is a better description than (1) in the tightbinding approximation, is derived by expanding the field operator in a Wannier basis of localized wave functions at each lattice site. This Hamiltonian has has three contributions: a kinetic energy term yielding contributions from tunnelling between adjacent wells, an energy offset in each lattice site (due, for example, to external confinement), and a potential energy term characterized by atom-atom interactions (that indicates how much energy it takes to put a second atom into a lattice site that already has one atom present). One can use the Bose-Hubbard Hamiltonian to examine transitions between superfluidity and Mott insulation. ${ }^{30}$

In the present paper, we examine in detail the band structure of BECs in shallow periodic lattices using Hamiltonian perturbation theory and supporting numerical simulations. ${ }^{44}$ Our methodology, which yields analytical expressions describing the features of BEC resonance bands, exploits the elliptic function solutions of the NLS in the absence of a potential. Note, however, that this paper does not explore the chaotic dynamics of BECs.

\section{Coherent Structures}

We consider uniformly propagating coherent structures with the ansatz $\psi(x-v t, t)=R(x-$ $v t) \exp (i[\theta(x-v t)-\mu t])$, where $R \equiv|\psi|$ is the magnitude (amplitude) of the wave function, $v$ is the velocity of the coherent structure, $\theta(x)$ determines its phase, $\vec{v}_{0} \propto \nabla \theta$ is the particle velocity, and $\mu$ is the chemical potential (which can be termed an angular frequency from a dynamical systems perspective). Considering a coordinate system that travels with speed $v$ (by defining $x^{\prime}=x-v t$ and relabeling $x^{\prime}$ as $x$ ) yields

$$
\psi(x, t)=R(x) \exp (i[\theta(x)-\mu t]) .
$$


[From a physical perspective, we consider the case $v=0$, as $V\left(x^{\prime}\right)=V(x-v t)$.] When the (temporally periodic) coherent structure (3) is also spatially periodic, it is called a modulated amplitude wave (MAW). ${ }^{12,13}$ The orbital stability of MAWS for the cubic NLS with elliptic potentials has been studied by Bronski and co-authors. ${ }^{9-11}$ To obtain stability information about the sinusoidal potentials we consider, one takes the limit as the elliptic modulus $k$ approaches zero. ${ }^{35,45}$

When $V(x)$ is periodic, the resulting MAWs generalize the Bloch modes that occur in the theory of linear systems with periodic potentials, as one is considering a nonlinear Floquet-Bloch theory rather than a linear one. ${ }^{5,8,20,37,46}$ In this paper, we employ phase space methods and perturbation theory to examine MAWs and their concomitant band structure.

The novelty of our work lies in its illumination of BEC band structure through the use of perturbation theory and supporting numerical simulations to examine $2 m^{\prime}: 1$ spatial subharmonic resonances in BECs in periodic lattices. Such resonances correspond to spatially periodic solutions $\psi$ of period $2 m^{\prime}$ and generalize the 'period doubled' states (in $|\psi|^{2}$ ) studied by Machholm, et al. ${ }^{38}$ which pertain to the experiments of Cataliotti, et al.. ${ }^{19}$

Previous theoretical work in this area has focused on different aspects of BEC band structure, such as loop structure ${ }^{26,39,52}$ and hysteresis. ${ }^{42}$ In contrast to the coherent structures we consider, these authors studied band structure using a Bloch wave ansatz. In our notation, they assumed a priori that $R(x)=R(x+2 \pi / \kappa)$ has the same periodicity of the underlying spatial lattice $V(x)$, whereas we have made no such assumption and instead use Hamiltonian perturbation theory to study the dynamical behavior of $R(x)$. Additionally, the analytical components of these works are confined to two-to-three Fourier mode truncations of the Bloch wave dynamics. $^{26,39,52}$

Inserting (3) into the NLS (10) and equating real and imaginary parts yields

$$
\begin{aligned}
\hbar \mu R(x)= & -\frac{\hbar^{2}}{2 m} R^{\prime \prime}(x) \\
& +\left[\frac{\hbar^{2}}{2 m}\left[\theta^{\prime}(x)\right]^{2}+g R^{2}(x)+V(x)\right] R(x),
\end{aligned}
$$

$$
0=\frac{\hbar^{2}}{2 m}\left[2 \theta^{\prime}(x) R^{\prime}(x)+\theta^{\prime \prime}(x) R(x)\right],
$$

which gives the following two-dimensional system of nonlinear ordinary differential equations:

$$
\begin{aligned}
& R^{\prime}=S, \\
& S^{\prime}=\frac{c^{2}}{R^{3}}-\frac{2 m \mu R}{\hbar}+\frac{2 m g}{\hbar^{2}} R^{3}+\frac{2 m}{\hbar^{2}} V(x) R .
\end{aligned}
$$

The parameter $c$ is defined via the relation

$$
\theta^{\prime}(x)=\frac{c}{R^{2}},
$$

and therefore plays the role of "angular momentum," as discussed by Bronski and coauthors. ${ }^{10}$ [Equation (6) is a statement of conservation of angular momentum.] Constant phase solutions, which constitute an important special case, satisfy $c=0$.

\section{BECs without an External Potential}

When $V(x) \equiv 0$, the dynamical system (5) is autonomous and hence integrable, as it is twodimensional. Its equilibria $\left(R_{*}, S_{*}\right)$ satisfy $S_{*}=0$ and either $R_{*}=0, c=0$ or

$$
g R^{6}-\hbar \mu R^{4}+\frac{\hbar^{2}}{2 m} c^{2}=0,
$$

which can be solved exactly because it is cubic in $R^{2}$. When $c=0$, one obtains $R_{*}= \pm \sqrt{\hbar \mu / g}$. One thus obtains equilibria at $\left(R_{*}, 0\right) \neq(0,0)$ for $g>0$ if $\mu>0$ and $g<0$ if $\mu<0$.

The eigenvalues of the equilibrium $\left(R_{*}, 0\right)$ satisfy

$$
\lambda^{2}=-\frac{3 c^{2}}{R_{*}^{4}}-\frac{2 m \mu}{\hbar}+\frac{6 m g}{\hbar^{2}} R_{*}^{2} .
$$

When $c=0$ and $R_{*}=0$, one obtains $\lambda=$ $\pm \sqrt{-2 m \mu / \hbar}$. Additionally, one obtains a center at $(0,0)$ when $\mu>0$ and a saddle when $\mu<0$. One also obtains saddles at the $R_{*} \neq 0$ equilibria for $g>0$ when $\mu>0$ and centers at those same locations for $g<0$ when $\mu<0$. These latter equilibria are surrounded by periodic orbits that satisfy $R \neq 0$. The possible qualitative dynamics (for $c=0$ ) are illustrated in Figure 1 and summarized in Table 1

To study the dependence of the wave number of periodic orbits (centered at the origin) of (5) on the amplitude $R$ when $V(x) \equiv 0$, we employ Lindstedt's $\operatorname{method}^{46}$ and consider null angular-momentum wave functions for the case $\mu>0$. We also assume $g=\varepsilon \bar{g}$, where $\varepsilon \ll 1$ and $\bar{g}=\mathcal{O}(1)$. The period of $R(x)$ is given by

$$
T=\frac{2 \pi}{\alpha}=2 \pi\left[1+\frac{3 g A^{2}}{8 \mu \hbar}\right]+\mathcal{O}\left(\varepsilon^{2}\right),
$$


where $R(\xi)=R_{0}(\xi)+\mathcal{O}(\varepsilon), \xi:=\alpha x, \alpha=1+\varepsilon \alpha_{1}+$ $\mathcal{O}\left(\varepsilon^{2}\right)$ is the wave number, $A:=R_{0}(0)$, and

$$
R_{0}(\xi)=A \cos \left(\sqrt{\frac{2 m \mu}{\hbar}} \xi\right)
$$

Note that all periodic orbits are centered at the origin when $\mu>0$. When $g<0$, the spatial period becomes smaller with increasing $A$. When $g>0$, the period becomes larger with increasing $A$. In the latter case, the wave number-amplitude relation holds only for solutions inside the separatrix, as the trajectories are unbounded outside the separatrix and hence not periodic.

Before deriving the wave number-amplitude relations when $V(x) \neq 0$, we comment briefly on the preceeding results. The spatial period for small $c \neq 0$ is similar to (9), but it cannot be estimated as easily because equation (5) now includes a term of order $\mathcal{O}\left(R^{-3}\right)$ with coefficient $c$. Although (9) can be computed exactly in terms of elliptic functions, here we are interested in elucidating the qualitative dynamics of the MAWs of interest as well as establishing the methodology to be employed in the presence of potentials $V(x)$. We will utilize elliptic function solutions in Section [5in our detailed study of band structure. ${ }^{44}$ The physical relevance of elliptic functions to BECs has been discussed by Carr and collaborators. ${ }^{16,17}$

\section{BECs in a Periodic Lattice}

To study the wave number-amplitude relations of periodic orbits in the presence of external potentials, we expand the spatial variable $x$ in multiple scales. We define "stretched space" $\xi:=\alpha x$ as in the integrable situation and "slow space" $\eta:=\varepsilon x$. We consider potentials of the form $V(x)=\varepsilon \bar{V}(\xi, \eta)$, where $\bar{V}(\xi, \eta)=\bar{V}_{0} \sin \left[\kappa\left(\xi-\xi_{0}\right)\right]+\bar{V}_{1}(\eta)$ and $\bar{V}_{1}$, which is of order $\mathcal{O}(1)$, is arbitrary but slowly varying. Cases of particular interest include $\bar{V}_{1}=0$ (periodic potential) and $\bar{V}_{1}=\tilde{V}_{1}\left(\eta-\eta_{0}\right)^{2}$ (superposition of periodic and harmonic potentials).

When $\kappa \neq \pm 2 \beta:= \pm 2 \sqrt{2 m \mu / \hbar}$, the equations of motion for the slow dynamics of (5) with $c=0$ are

$$
\begin{aligned}
& \frac{d A}{d \eta} \equiv A^{\prime}=-\beta \alpha_{1} B-\frac{3 \beta \bar{g}}{8 \mu \hbar} B C^{2}-\frac{\beta}{2 \mu \hbar} B \bar{V}_{1}(\eta), \\
& \frac{d B}{d \eta} \equiv B^{\prime}=\beta \alpha_{1} A+\frac{3 \beta \bar{g}}{8 \mu \hbar} A C^{2}+\frac{\beta}{2 \mu \hbar} A \bar{V}_{1}(\eta),
\end{aligned}
$$

where $g \equiv \varepsilon \bar{g}$. The leading-order expression for the amplitude is

$$
R_{0}(\xi, \eta)=A(\eta) \cos (\beta \xi)+B(\eta) \sin (\beta \xi),
$$

where $C^{2}:=A(\eta)^{2}+B(\eta)^{2}$ is a constant. The dynamical system (11) is autonomous when $V_{1}(x) \equiv 0$. Equilibria $\left(A_{*}, B_{*}\right) \neq(0,0)$ of (11) correspond to periodic orbits of (5) with $c=0$. The equilibrium value of the squared amplitude is denoted $C_{*}^{2}=A_{*}^{2}+B_{*}^{2}$.

Converting to polar coordinates with $A(\eta)=$ $C \cos (\phi(\eta))$ and $B(\eta)=C \sin (\phi(\eta))$ and integrating the resulting equation yields

$$
\phi(\eta)=\phi(0)+\left[\alpha_{1} \beta+\frac{3 \beta \bar{g}}{8 \mu \hbar} C^{2}\right] \eta+\frac{\beta}{2 \mu \hbar} \int \bar{V}_{1}(\eta) d \eta .
$$

The wave number of the periodic motion is given by

$$
\alpha(C)=1-\frac{3 g}{8 \mu \hbar} C^{2}-\frac{1}{2 \mu \hbar} V_{1}(x)+\mathcal{O}\left(\varepsilon^{2}\right) .
$$

When $\kappa= \pm 2 \beta$, we show that the slow flow equations have an extra term due to resonance. Without loss of generality, we let $\kappa=+2 \beta$, as changing the sign of $V_{0}$ produces the $\kappa=-2 \beta$ case. When $m=0.5, \mu=10$, and $\hbar=1$, for example, one obtains this resonant situation for $\kappa= \pm 10$. Additionally, we show that $C$ is no longer constant in this resonant situation.

In polar coordinates, the slow flow equations are

$\phi^{\prime}=\alpha_{1} \beta+\frac{3 \beta \bar{g}}{8 \mu \hbar} C^{2}+\frac{\bar{V}_{0} \beta}{4 \mu \hbar} \sin \left[2\left(\phi-\beta \xi_{0}\right)\right]+\frac{\beta}{2 \mu \hbar} \bar{V}_{1}(\eta)$,

$C^{\prime}=-\frac{\bar{V}_{0} \beta}{4 \mu \hbar} C \cos \left[2\left(\phi-2 \beta \xi_{0}\right)\right]$

Integrating the equation for $C^{\prime}$ yields

$$
C=C_{0} \exp \left[-\frac{\bar{V}_{0} \beta}{4 \mu \hbar} \int \cos \left[2\left(\phi(\eta)-\beta \xi_{0}\right)\right] d \eta\right],
$$

which one may then insert into the equation for the angular dynamics.

To determine equilibria, one puts $C^{\prime}=\phi^{\prime}=0$. From $C^{\prime}=0$, one determines that equilibria $\left(C_{*}, \phi_{*}\right)$ satisfy

$$
\phi_{*}=\frac{(2 j+1) \pi}{4}+\beta \xi_{0}, \quad j \in\{0,1,2,3\},
$$

which is independent of the scattering coefficient. Inserting (17) into $C^{\prime}=0$ yields the wave numberamplitude relation

$$
\alpha_{R}(C)=\alpha(C) \mp \frac{V_{0}}{4 \mu \hbar}+\mathcal{O}\left(\varepsilon^{2}\right),
$$

for periodic orbits of (5). In (18), the minus sign is obtained when $j \in\{0,2\}$, and the plus sign is obtained when $j \in\{1,3\}$. Equation (18) is valid for $2: 1$ 
spatial resonances. We examine $2 m^{\prime}: 1$ resonances for integer $m^{\prime}$ in Section [5] using Hamiltonian perturbation theory and the elliptic function solutions of (5) when $V_{0}=0$.

To examine the spatial stability (i.e., stability with respect to spatial evolution) of these periodic orbits in the presence of resonant periodic potentials, we compute the spatial stability of equilibria of (15) when $V_{1}(x) \equiv 0$. The eigenvalues of the periodic orbits are

$$
\lambda= \pm \frac{\beta}{2 \mu} \sqrt{\mp \frac{3 \bar{V}_{0} \bar{g} C}{2 \hbar^{2}}} .
$$

We show numerical simulations for (5) in the presence of a periodic potential in Figure 2 In this situation, (5) is a nonlinear Mathieu equation. ${ }^{45,46,53}$ Figure 2 shows the coherent structure for the trajectory with $\kappa=100$ and $V_{0}=10$. Figure 3 depicts a Poincaré section describing the dynamics of ${ }^{85} R b$, for which $a=-0.9 \mathrm{~nm}$. Figure 4 depicts spatial profiles of the coherent structures corresponding to the locally chaotic and globally chaotic trajectories in Figure 3

\section{Subharmonic Resonances}

In this section, we analyze spatial subharmonic resonances and the band structure of repulsive BECs with a positive chemical potential. We perturb off the elliptic function solutions of the underlying integrable system in order to study $2 m^{\prime}: 1$ spatial resonances with a leading-order perturbation method. Perturbing off simple harmonic functions, by contrast, requires a perturbative method of order $m^{\prime}$ to study $2 m^{\prime}: 1$ resonances. At the center of the KAM islands, we observe 'period-multiplied' states. When $m^{\prime}=1$, one obtains period-doubled states in $\psi$. As verified numerically in Section 6] our qualitative results are excellent. Given that our method is a leading-order one, our quantitative results are also remarkably good.

Recent work by Machholm and coauthors ${ }^{38}$ on period-doubled states (in $|\psi|^{2}$ ) follows up experimental studies by Cataliotii and coauthors, ${ }^{19}$ who observed superfluid current disruption in chains of weakly coupled BECs, which is related to the dependence of the dynamical instability of Bloch states on the magnitude of particle interactions. Perioddoubled states, which may be interpreted as soliton trains, arise from dynamical instabilities of the energy bands associated with Bloch states. ${ }^{38}$ In the present work, we offer a dynamical systems perspective on period-doubled states and their generaliza- tions. Our theoretical and computational analysis reveals period-multiplied solutions of the GP (1). The existence of these wave functions can be explored experimentally.

A detailed examination of the band structure of BECs in periodic lattices requires a more intricate perturbative analysis than that discussed earlier in this work. Previous authors have concentrated on numerical studies of band structure. ${ }^{8,20,37}$ The approach we take, on the other hand, is to analyze the spatial resonance structure that arises from the nonlinear Mathieu equation obtained upon the application of a coherent structure ansatz to the cubic NLS. We examine situations with null angular momentum $(c=0)$, but one observes similar behavior when $c \neq 0$ when $R$ is away from the origin. The analytical approach we employ was introduced by Zounes and Rand ${ }^{53}$ for $g<0$ and $\mu>0$ (see Figure 1b), the technically easiest case to consider. Their study of nonlinear Mathieu equations is directly applicable to BECs. Our work is an extension of their work to the situation $g>0, \mu>0$ (see Figure [a), the second easiest case to consider. We study this case in detail and also apply the results of Zounes and Rand to attractive BECs with a positive chemical potential. We briefly discuss attractive BECs with a negative chemical potential (see Figure 1r), the technically hardest case to consider. Note that this paper does not explore the chaotic dynamics of BECs, which is an important open issue. $31,45,46,51$

$$
\begin{gathered}
\text { Let } x_{0}=-\pi /(2 \kappa) \text { and } V_{1}(x) \equiv 0 \text { so that } \\
\qquad V(x)=V_{0} \cos (\kappa x) .
\end{gathered}
$$

When $c=0$, the equations of motion (2), (5) for the amplitude of the coherent structure (3) take the form

$$
R^{\prime \prime}+\delta R+\alpha R^{3}+\epsilon R \cos (\kappa x)=0,
$$

where

$$
\delta=\frac{2 m \mu}{\hbar}, \quad \alpha=-\frac{2 m g}{\hbar^{2}}, \quad \epsilon=-\frac{2 m}{\hbar^{2}} V_{0} .
$$

(Note that the perturbation parameter $\epsilon$ is not the same as the parameter $\varepsilon$ employed earlier.) The parameters $\mu, V_{0}, \kappa$, and $a$ (and hence $g$ ) can all be adjusted experimentally. When $\epsilon=0$, solutions of (21) can be written exactly in terms of elliptic functions: ${ }^{21,22,35,45,50,53}$

$$
R=\sigma \rho \operatorname{cn}(u, k),
$$


where

$$
\begin{aligned}
u & =u_{1} x+u_{0}, \quad u_{1}^{2}=\delta+\alpha \rho^{2}, \\
k^{2} & =\frac{\alpha \rho^{2}}{2\left(\delta+\alpha \rho^{2}\right)}, \\
u_{1} & \geq 0, \quad \rho \geq 0, \quad k^{2} \in \mathbb{R}, \quad \sigma \in\{-1,1\},
\end{aligned}
$$

and $u_{0}$ is obtained from an initial condition (and can be set to 0 without loss of generality). We consider $u_{1} \in \mathbb{R}$ in order to study periodic solutions. One can use argument transformations to study solutions with complex $u_{1}$. When $k^{2} \in(1, \infty)$, one makes sense of the cn function with a reciprocal modulus transformation. ${ }^{21,22}$ When $k^{2}<0$, one employs a reciprocal complementary modulus transformation, which we discuss below.

Equation (21) can be integrated when $\epsilon=0$ to yield the Hamiltonian

$$
\frac{1}{2} R^{\prime 2}+\frac{1}{2} \delta R^{2}+\frac{1}{4} \alpha R^{4}=h
$$

with given energy $h$. With (24), one computes

$$
h=\frac{1}{4} \rho^{2}\left(2 \delta+\alpha \rho^{2}\right)=\frac{\delta^{2}}{\alpha} \frac{k^{2} k^{\prime 2}}{\left(1-2 k^{2}\right)^{2}},
$$

where $k^{\prime 2}:=1-k^{2}$. Earlier in this paper, we enumerated the different possibilities for the qualitative dynamics of (21) in terms of the signs of $\mu$ and $g$ (and hence in terms of the signs of $\delta$ and $\alpha$ ).

\subsection{Repulsive BECs with a Positive Chemical Potential}

We first consider in detail the case $g>0, \mu>0$, for which $\delta>0, \alpha<0$. For notational convenience, we sometimes utilize $\alpha^{\prime}:=-\alpha$. This analysis involves a considerable amount of elliptic-function manipulation, but we are rewarded in the end by a much more effective perturbation theory than can be obtained by employing trigonometric functions.

The center at $(0,0)$ satisfies $h=\rho^{2}=k^{2}=0$. The saddles at $\left( \pm \sqrt{\delta / \alpha^{\prime}}, 0\right)$ and their adjoining separatrix satisfy

$$
h=-\frac{\delta^{2}}{4 \alpha}, \quad \rho^{2}=\frac{\delta}{|\alpha|}, \quad k^{2}=-\infty .
$$

The $\operatorname{sign} \sigma=+1$ is used for the right saddle, and $\sigma=$ -1 is used for the left one. Within the separatrix, all orbits are periodic and the value of $\sigma$ is immaterial.

\subsubsection{Action-Angle Variable Description and Transformations}

For this choice of parameters, $k^{2} \in[-\infty, 0]$, so elliptic functions are defined through the reciprocal complementary modulus transformation, ${ }^{21,22}$ which relates the $(u, k)$ coordinate system to another coordinate system, which we denote $\left(w, k_{2}\right)$. To tranform between these two coordinate systems, one uses the following relations:

$$
\begin{aligned}
\operatorname{cn}(u, k) & =\operatorname{cd}\left(w, k_{2}\right), \\
\operatorname{dn}(u, k) & =\operatorname{nd}\left(w, k_{2}\right), \\
\operatorname{sn}(u, k) & =k_{2}^{\prime} \operatorname{sd}\left(w, k_{2}\right), \\
k^{\prime}=\frac{1}{k_{2}^{\prime}}, u & =k_{2}^{\prime} w, K=k_{2}^{\prime} K_{2}, E=\frac{1}{k_{2}^{\prime}} E_{2} .
\end{aligned}
$$

Here, $K \equiv K(k)$ denotes the complete elliptic integral of the first kind, $E \equiv E(k)$ denotes the complete elliptic integral of the second kind, and items with the subscript ' 2 ' denote the analogous quantities in the $\left(w, k_{2}\right)$ coordinate system. ${ }^{21,35,50}$

We rescale (21) using the coordinate transformation

$$
\chi=\sqrt{\delta} x, \quad r=\sqrt{\frac{\delta}{\alpha^{\prime}}} R
$$

to obtain

$$
r^{\prime \prime}+r-r^{3}=0
$$

when $V(x) \equiv 0$. (Note that in this analysis, the quantity $\chi$ does not represent the mean healing length.) In terms of the original coordinates,

$$
R(x)=\sqrt{\frac{\delta}{\alpha^{\prime}}} r(\sqrt{\delta} x)=\sqrt{\frac{\hbar \mu}{g}} r\left(\sqrt{\frac{2 m \mu}{\hbar}} x\right) .
$$

The rescaling applied for other choices of $\delta$ and $\alpha$ differ slightly from that in (29), so that the arguments of their associated square roots are positive.

The Hamiltonian corresponding to (30) is

$$
H_{0}(r, s)=\frac{1}{2} s^{2}+\frac{1}{2} r^{2}-\frac{1}{4} r^{4}=h, h \in[0,1 / 4],
$$

where $s:=r^{\prime}=d r / d \chi$. Additionally, $\rho^{2} \in[0,1]$, $k_{2}^{2} \in[0,1]$ (corresponding to $k^{2} \in(-\infty, 0]$ in the original coordinates), and

$$
k^{2}=\frac{\rho^{2}}{2\left(\rho^{2}-1\right)} .
$$

With the initial condition $r(0)=\rho, s(0)=0$, which 
implies that $u_{0}=0$, solutions to (30) are given by

$$
\begin{aligned}
r(\chi)=\rho \operatorname{cn}\left(\left[1-\rho^{2}\right]^{1 / 2} \chi, k\right) & \\
s(\chi)=- & \rho\left[1-\rho^{2}\right]^{1 / 2} \operatorname{sn}\left(\left[1-\rho^{2}\right]^{1 / 2} \chi, k\right) \\
& \times \operatorname{dn}\left(\left[1-\rho^{2}\right]^{1 / 2} \chi, k\right) .
\end{aligned}
$$

The period of a given periodic orbit $\Gamma$ is

$$
T(k)=\oint_{\Gamma} d \chi=\frac{4 K(k)}{\sqrt{1-\rho^{2}}},
$$

where $4 K(k)$ is the period in $u$ of $\operatorname{cn}(u, k) .{ }^{50}$ The frequency of this orbit is

$$
\Omega(k)=\frac{\pi \sqrt{1-\rho^{2}}}{2 K(k)} .
$$

Let $\Gamma_{h}$ denote the periodic orbit with energy $h=$ $H_{0}(r, s)$. The area of phase space enclosed by this orbit is constant with respect to $\chi$, so one may define the action ${ }^{21,22,29,45}$

$$
J:=\frac{1}{2 \pi} \oint_{\Gamma_{h}} s d r=\frac{1}{2 \pi} \int_{0}^{T(k)}[s(\chi)]^{2} d \chi,
$$

which in this case can be evaluated exactly:

$$
J=\frac{4 \sqrt{1-\rho^{2}}}{3 \pi}\left[E(k)-\left(1-\rho^{2} / 2\right) K(k)\right] .
$$

The associated angle ${ }^{29,31,36,51}$ in the canonical transformation $(r, s) \longrightarrow(J, \Phi)$ is

$$
\Phi:=\Phi(0)+\Omega(k) \chi .
$$

The frequency $\Omega(k)$ monotonically decreases as $k^{2}$ goes from $-\infty$ to 0 [that is, as one goes from the separatrix to the center at $(r, s)=(0,0)]$. With this transformation, equation (34) becomes

$$
\begin{aligned}
r(J, \Phi)= & \rho(J) \operatorname{cn}(2 K(k) \Phi / \pi, k) \\
s(\chi)=- & \rho(J) \sqrt{1-\rho(J)^{2}} \operatorname{sn}(2 K(k) \Phi / \pi, k) \\
& \times \operatorname{dn}(2 K(k) \Phi / \pi, k),
\end{aligned}
$$

where $k=k(J)$.

After rescaling, the equations of motion for the forced system (21) take the form

$$
r^{\prime \prime}+r-r^{3}+\frac{\epsilon}{\delta} \cos \left(\frac{\kappa}{\sqrt{\delta}} \chi\right) r=0
$$

with the corresponding Hamiltonian

$$
\begin{aligned}
H(r, s, \chi) & =H_{0}(r, s)+\epsilon H_{1}(r, s, \chi) \\
& =\frac{1}{2} s^{2}+\frac{1}{2} r^{2}-\frac{1}{4} r^{4}+\frac{\epsilon}{2 \delta} r^{2} \cos \left(\frac{\kappa}{\sqrt{\delta}} \chi\right) .
\end{aligned}
$$

In action-angle coordinates, this becomes

$$
\begin{aligned}
H(\Phi, J, \chi) & =h(J)+\epsilon h_{1}(\Phi, J, \chi) \\
& =\frac{1}{2} \rho(J)^{2}-\frac{1}{4} \rho(J)^{4} \\
& +\frac{\epsilon}{2 \delta} \rho(J)^{2} \operatorname{cn}^{2}(2 K(k) \Phi / \pi, k) \cos \left(\frac{\kappa}{\sqrt{\delta}} \chi\right) .
\end{aligned}
$$

One obtains a second action-angle pair $(\phi, j)$ using the canonical transformation $(\Phi, J) \longrightarrow(\phi, j)$ defined by the relations

$$
j(J)=\frac{1}{2} \rho(J)^{2}, \quad \Phi(\phi, j)=\frac{\phi}{J^{\prime}(j)},
$$

where

$$
\begin{aligned}
k^{2} & =\frac{j}{2 j-1}, \\
J(j) & =\frac{2}{3} \sqrt{1-2 j}[\tilde{E}(j)-(1-j) \tilde{K}(j)], \\
\tilde{K}(j) & =\frac{2}{\pi} K[k(j)], \quad \tilde{E}(j)=\frac{2}{\pi} E[k(j)] .
\end{aligned}
$$

Additionally,

$$
J^{\prime}(j):=\frac{d J}{d j}=\sqrt{1-2 j} \tilde{K}(j)=\frac{1-2 j}{\Omega(j)} .
$$

Note that $J \sim j$ for small-amplitude motion. Furthermore, $j=0$ at the origin, and $j=1 / 2$ on the separatrix.

The Hamiltonian (43) becomes

$$
\begin{aligned}
H(\phi, j, \chi) & =H_{0}(j)+\epsilon H_{1}(\phi, j, \chi) \\
& =j-j^{2}+\frac{\epsilon}{\delta} j \mathrm{cn}^{2}\left(\frac{\tilde{K}(j)}{J^{\prime}(j)} \phi, k\right) \cos \left(\frac{\kappa}{\sqrt{\delta}} \chi\right) .
\end{aligned}
$$

Because we have used elliptic functions rather than trigonometric functions, all results are exact thus far. ${ }^{53}$

\subsubsection{Perturbative Analysis}

A subsequent $\mathcal{O}(\epsilon)$ analysis at this stage allows one to study $2 m^{\prime}: 1$ subharmonic resonances for all $m^{\prime} \in \mathbb{Z}$. By contrast, had we undertaken this procedure with 
trigonometric functions (which would have entailed a perturbative approach from the beginning), an $\mathcal{O}\left(\epsilon^{m^{\prime}}\right)$ analysis would be required to study $2 m^{\prime}: 1$ subharmonic resonances of (21).

The Fourier expansion of $\mathrm{cn}$ is given by

$$
\operatorname{cn}(u, k)=\frac{2 \pi}{k K(k)} \sum_{n=0}^{\infty} b_{n}(k) \cos \left[(2 n+1) \frac{\pi u}{2 K(k)}\right]
$$

where the Fourier coefficients $b_{n}(k)$ are

$$
b_{n}(k)=\frac{1}{2} \operatorname{sech}\left[(n+1 / 2) \pi K^{\prime}(k) / K(k)\right]
$$

and $K^{\prime}(k):=K\left(\sqrt{1-k^{2}}\right)$ denotes the complementary complete elliptic integral of the first kind. $1,50,53$ In the present situation,

$$
\operatorname{cn}\left(\frac{\tilde{K}(j)}{J^{\prime}(j)} \phi, k\right)=\sum_{n=0}^{\infty} B_{n}(j) \cos \left[(2 n+1) \frac{\phi}{J^{\prime}(j)}\right]
$$

where

$$
B_{n}(j)=\frac{4}{k(j) \tilde{K}(j)} b_{n}[k(j)] .
$$

Consequently,

$$
\operatorname{cn}^{2}\left(\frac{\tilde{K}(j)}{J^{\prime}(j)} \phi, k\right)=\mathcal{B}_{0}(j)+\sum_{l=1}^{\infty} \mathcal{B}_{l} \cos \left(\frac{2 l \phi}{J^{\prime}(j)}\right)
$$

where the Fourier coefficients $\mathcal{B}_{l}(j)$ are obtained by convolving the previous Fourier coefficients (51) with each other. ${ }^{53}$

Before proceeding, it is important to discuss the computation of the coefficients $\mathcal{B}_{l}(j)$, which require some care. Using the Elliptic Nome ${ }^{1,54}$

$$
q(k):=e^{-\pi K^{\prime}(k) / K(k)},
$$

the Fourier coefficient (49) is expressed as

$$
b_{n}(k)=\frac{1}{q(k)^{n+1 / 2}+q(k)^{-(n+1 / 2)}} .
$$

One then expands $\mathcal{B}_{l}(j)$ in Taylor series about $j=0$. In this computation, one finds that the coefficients of even powers of $j$ in $\mathcal{B}_{l}(j)$ are the same as when $g<0$, $\mu>0$ and that odd powers have the opposite sign. This distinction lies at the root of the qualitatively different dynamics in the two cases, which we will discuss in Section 5.2 Recall that their underlying integrable dynamics are depicted in Figure 1
After the Fourier expansion, the perturbative term in the Hamiltonian (47) is

$$
\begin{aligned}
H_{1}(\phi, j, \chi)= & \frac{1}{\delta} \mathcal{B}_{0}(j) \cos \left(\frac{\kappa}{\sqrt{\delta}} \chi\right) \\
+ & \frac{1}{2 \delta} \sum_{l=1}^{\infty} \mathcal{B}_{l}(j)\left[\cos \left(\frac{2 l \phi}{J^{\prime}(j)}+\frac{\kappa}{\sqrt{\delta}} \chi\right)\right. \\
& \left.+\cos \left(\frac{2 l \phi}{J^{\prime}(j)}-\frac{\kappa}{\sqrt{\delta}} \chi\right)\right] .
\end{aligned}
$$

There are infinitely many (subharmonic) resonance bands, ${ }^{31,45,51}$ each of which corresponds to a single harmonic in the perturbation series (55). To isolate individual resonances, we apply a canonical, nearidentity transformation $29,31,45,51,53$ to the Hamiltonian $H=H_{0}+\epsilon H_{1}$. This transformation is given by

$$
\begin{aligned}
& \phi=Q+\epsilon \frac{\partial W_{1}}{\partial P}+\mathcal{O}\left(\epsilon^{2}\right), \\
& j=P-\epsilon \frac{\partial W_{1}}{\partial Q}+\mathcal{O}\left(\epsilon^{2}\right),
\end{aligned}
$$

where the generating function $W_{1}$ is

$$
\begin{aligned}
W_{1}=\frac{P \mathcal{B}_{0}(P)}{\kappa \sqrt{\delta}} \sin \left(\frac{\kappa}{\sqrt{\delta}} \chi\right) \\
+\frac{P}{2 \sqrt{\delta}} \sum_{l=1, l \neq m^{\prime}}^{\infty} \mathcal{B}_{l}(P)\left[\frac{\sin \left(\frac{2 l Q}{J^{\prime}(P)}+\frac{\kappa}{\sqrt{\delta}} \chi\right)}{\kappa+2 l \sqrt{\delta} \Omega(P)}\right. \\
\left.+\frac{\sin \left(\frac{2 l Q}{J^{\prime}(P)}-\frac{\kappa}{\sqrt{\delta}} \chi\right)}{\kappa-2 l \sqrt{\delta} \Omega(P)}\right] .
\end{aligned}
$$

To obtain (57), one uses the fact [from (46)] that $\Omega(P)=(1-2 P) / J^{\prime}(P)$.

The resulting Hamiltonian is

$$
\begin{aligned}
K(Q, P, \chi) & =K_{0}(P)+\epsilon K_{1}(Q, P, \chi), \\
K_{0}(P) & =P-P^{2}=H_{0}(P), \\
K_{1}(Q, P, \chi) & =H_{1}(Q, P, \chi)+\left\{H_{0}, W_{1}\right\}-\frac{\partial W_{1}}{\partial \chi},
\end{aligned}
$$

where $\left\{A_{1}, A_{2}\right\}$ denotes the Poisson bracket of $A_{1}$ and $A_{2}$. For the present choice of $W_{1}$, one obtains the resonance Hamiltonian

$$
\begin{aligned}
& K\left(Q, P, \chi ; m^{\prime}\right)=P-P^{2} \\
& \quad+\frac{\epsilon}{2 \delta} P \mathcal{B}_{m^{\prime}}(P) \cos \left(\frac{2 m^{\prime} Q}{J^{\prime}(P)}-\frac{\kappa}{\sqrt{\delta}} \chi\right)+\mathcal{O}\left(\epsilon^{2}\right) .
\end{aligned}
$$


The choice of the generating function (57) eliminates all resonances from the Hamiltonian $K$ except the $2 m^{\prime}: 1$ resonance. In focusing on a single resonance band in phase space, one restricts $P$ to a neighborhood of $P_{m^{\prime}}$, which denotes the location of the $m^{\prime}$ th resonant torus (associated with periodic orbits in $2 m^{\prime}: 1$ spatial resonance with the periodic lattice).

\subsubsection{Resonance Relations}

Resonant frequencies arise when the denominators of the terms in $W_{1}$ vanish, ${ }^{31,45,53}$ which yields the equation

$$
\frac{\kappa}{\sqrt{\delta}}= \pm 2 m^{\prime} \Omega\left(P_{m}\right)
$$

for the resonance of order $2 m^{\prime}: 1$. As $\Omega \leq 1$ is a decreasing function of $P \in[0,1 / 2)$, the resonance band associated with $2 m^{\prime}: 1$ subharmonic spatial resonances is present when

$$
\frac{\kappa}{\sqrt{\delta}} \leq 2 m^{\prime}
$$

For example, when $\kappa=2.5$ and $\delta=1$, there are resonances of order $4: 1,6: 1,8: 1$, etc, but there are no resonances or order $2: 1$. When $\kappa=5$ and $\delta=1$, there are resonances of order $6: 1,8: 1,10: 1$, etc, but there are no resonances of order $2: 1$ or $4: 1$. In terms of the original parameters, the condition (61) describing the onset of $2 m^{\prime}: 1$ resonance bands takes the form

$$
\kappa \leq 2 m^{\prime} \sqrt{\frac{2 m \mu}{\hbar}} .
$$

If the lattice $V(x)$ has a smaller wave number (larger periodicity), then the chemical potential $\mu$ must be smaller for a given resonance to occur. As $\kappa$ is decreased for a fixed $\mu$ (i.e., $\delta$ ) or as $\mu$ is increased for a given lattice size $\kappa$, resonance bands of lower order emerge from the origin and propagate in phase space. Consequently, a sufficiently high order resonance is always present in (21), but a given number of low-order ones may not be. Lower-order resonances occupy larger regions of phase space, so (61) also indicates the volume of phase space affected by spatial resonances. We will illustrate this in more detail in Section [6] with numerical simulations.

\subsubsection{Analytical Description of Resonance Bands}

To further examine the resonance structure of (21), we make (59) autonomous via another canonical change of coordinates. ${ }^{45,53}$ Toward this end, we define the generating function

$$
F\left(Q, Y, \chi ; m^{\prime}\right)=Q Y-\frac{\kappa}{2 m^{\prime} \sqrt{\delta}} J(Y) \chi
$$

which yields

$$
\begin{aligned}
P & =\frac{\partial F}{\partial Q}(Q, Y, \chi)=Y \\
\xi & =\frac{\partial F}{\partial Y}(Q, Y, \chi) \\
& =Q-\frac{\kappa}{2 m^{\prime} \sqrt{\delta}} J^{\prime}(Y) \chi=Q-\frac{\kappa}{2 m^{\prime} \sqrt{\delta}} J^{\prime}(P) \chi .
\end{aligned}
$$

(Note that in this analysis, $\xi$ does not represent stretched space, as it did in our multiple scale expansion.) With this final transformation, the resonance Hamiltonian (59) becomes

$$
\begin{aligned}
K_{m^{\prime}}(\xi, Y)= & K\left(Q, P, \chi ; m^{\prime}\right)+\frac{\partial F}{\partial \chi}(Q, Y, \chi) \\
=Y & -Y^{2}-\frac{\kappa}{2 m^{\prime} \sqrt{\delta}} J(Y) \\
& +\frac{\epsilon}{2 \delta} Y \mathcal{B}_{m^{\prime}}(Y) \cos \left(\frac{2 m^{\prime} \xi}{J^{\prime}(Y)}\right),
\end{aligned}
$$

which is integrable in the $(Y, \xi)$ coordinate system. In $(R, S)$-space, level curves of $K_{m^{\prime}}$ correspond to invariant curves of Poincaré sections of (21), which are defined by strobing the system when the spatial variable takes the values $x_{n}=2 n \pi / \kappa$.

We now provide an analytical description of the resonance bands under discussion. In particular, we compute the locations and type of equilibria and width of resonance bands as functions of the parameters $\delta, \epsilon$, and $\kappa$, and hence of $\mu, V_{0}$, and $\kappa$. Such bands emerge from the action $P=Y=Y_{m^{\prime}}$, which designates the location of the $m^{\prime}$ th resonance torus in phase space and is determined by the resonance relation (60):

$$
\frac{\kappa}{\sqrt{\delta}}=2 m^{\prime} \Omega\left(Y_{m^{\prime}}\right)
$$

This resonance band is associated with periodic orbits in $2 m^{\prime}: 1$ spatial resonance with the periodic lattice. The resonance torus is filled with degenerate periodic orbits that split ${ }^{31,51}$ into $2 m^{\prime}$ saddles and $2 m^{\prime}$ centers when a perturbation is introduced. 
From (65), one obtains Hamilton's equations

$$
\begin{aligned}
& Y^{\prime}=- \frac{\partial K_{m^{\prime}}}{\partial \xi}=\frac{\epsilon Y \mathcal{B}_{m^{\prime}}(Y)}{J^{\prime}(Y)} \sin \left(\frac{2 m^{\prime} \xi}{J^{\prime}(Y)}\right) \\
& \xi^{\prime}=\frac{\partial K_{m^{\prime}}}{\partial Y}= 1-2 Y-\frac{\kappa}{2 m^{\prime} \sqrt{\delta}} J^{\prime}(Y) \\
&+\frac{\epsilon}{2 \delta}\left[\left(Y \mathcal{B}_{m^{\prime}}(Y)\right)^{\prime} \cos \left(\frac{2 m^{\prime} \xi}{J^{\prime}(Y)}\right)\right. \\
&\left.+2 m^{\prime} \xi \frac{J^{\prime \prime}(Y)}{\left[J^{\prime}(Y)\right]^{2}} Y \mathcal{B}_{m^{\prime}}(Y) \sin \left(\frac{2 m^{\prime} \xi}{J^{\prime}(Y)}\right)\right] .
\end{aligned}
$$

Equilibria satisfy either $Y=0$ or

$$
\sin \left(\frac{2 m^{\prime} \xi}{J^{\prime}(Y)}\right)=0 .
$$

They also satisfy

$$
\xi^{\prime}=0=1-2 Y-\frac{\kappa}{2 m^{\prime} \sqrt{\delta}} J^{\prime}(Y) \pm \frac{\epsilon}{2 \delta}\left[Y \mathcal{B}_{m^{\prime}}(Y)\right]^{\prime}
$$

where the sign \pm in (69) arises from

$$
\cos \left(\frac{2 m^{\prime} \xi}{J^{\prime}(Y)}\right)=0 .
$$

Using $J^{\prime}(Y)=\sqrt{1-2 Y} \tilde{K}(Y)$, equation (69) is written

$1-2 Y-\frac{\kappa}{2 m^{\prime} \sqrt{\delta}} \sqrt{1-2 Y} \tilde{K}(Y) \pm \frac{\epsilon}{2 \delta}\left[Y \mathcal{B}_{m^{\prime}}(Y)\right]^{\prime}=0$.

When $g<0$ and $\mu>0$, the + case yields a saddle and the - case yields a center. In the present situation $(g>0, \mu>0)$, this holds for odd $m^{\prime}$. When $m^{\prime}$ is even, - is a saddle and + is a center.

At equilibria, the action $Y$ takes the value

$$
Y_{e}=Y_{m^{\prime}}+\epsilon \Delta Y+\mathcal{O}\left(\epsilon^{2}\right)=Y_{m^{\prime}} \pm \mathcal{O}(\epsilon)
$$

with the signs as in (71). However, note that $Y_{c}>$ $Y_{m^{\prime}}>Y_{s}$, just as for $g<0$. One inserts (72) into (71) and expands the result in a power series. At order $\mathcal{O}\left(\epsilon^{0}\right)=\mathcal{O}(1)$, this reproduces the resonance relation (66). At order $\mathcal{O}(\epsilon)$, one obtains

$$
\Delta Y=\mp \frac{\epsilon}{2 \delta}\left[\frac{\mathcal{B}_{m^{\prime}}\left(Y_{m^{\prime}}\right)+Y_{m^{\prime}} \mathcal{B}_{m^{\prime}}^{\prime}\left(Y_{m^{\prime}}\right)}{\Omega\left(Y_{m^{\prime}}\right) \sqrt{1-2 Y_{m^{\prime}}} \tilde{K}^{\prime}\left(Y_{m^{\prime}}\right)-1}\right],
$$

where saddles $Y_{s}$ use the + sign and centers $Y_{c}$ use the $-\operatorname{sign}$ when $m^{\prime}$ is even, and the opposite is true when $m^{\prime}$ is odd. When $m^{\prime}$ is even, $\Delta Y>0$, but
$\Delta Y<0$ when $m^{\prime}$ is odd. Additionally, $Y_{c}$ is always larger than $Y_{s}$ (for both signs of $\epsilon$ ).

Resonance bands occupy a finite region of phase space bounded by a pendulum-like separatrix. When a perturbation is introduced, trajectories outside the separatrix behave almost as they would in the absence of a perturbation, so it is important to estimate the width of resonance bands, which emerge at action values satisfying the resonance relation (66). Because of the direction of the inequality in (62), this is more of a condition for non-existence of given resonances. [See the discussion following equation (61).] For a given set of parameters, there will always be resonances of sufficiently high order (i.e., for a sufficiently large $m^{\prime}$ ). However, as we illustrate numerically below, there are parameter regions in which no $2: 1$ resonances exist, regions in which no $2: 1$ or $4: 1$ resonances exist, etc. This behavior contrasts markedly with that observed when $g<0 .{ }^{53}$ In that situation, there exist parameter regions in which only $2: 1$ resonances exist, regions in which only $2: 1$ and $4: 1$ resonances exist, etc.

We now show that the width of a resonance band is

$$
\mathcal{O}\left(\sqrt{\frac{Y_{m^{\prime}} \mathcal{B}_{m^{\prime}}\left(Y_{m^{\prime}}\right)|\epsilon|}{\delta}}\right)
$$

for perturbations of size $\epsilon=-2 m V_{0} / \hbar^{2}$.

The separatrix of interest passes through the saddle point $Y_{s}$, and the maximum extent of the resonance band occurs at the same phase $\xi$ as the associated center, so

$$
\begin{array}{r}
K_{m^{\prime}}\left(\cos \left(\frac{2 m^{\prime} \xi}{J^{\prime}\left(Y_{s}\right)}\right)=-1, Y=Y_{s}\right) \\
=K_{m^{\prime}}\left(\cos \left(\frac{2 m^{\prime} \xi}{J^{\prime}(Y)}\right)=+1, Y\right)
\end{array}
$$

when $m^{\prime}$ is odd and

$$
\begin{array}{r}
K_{m^{\prime}}\left(\cos \left(\frac{2 m^{\prime} \xi}{J^{\prime}\left(Y_{s}\right)}\right)=+1, Y=Y_{s}\right) \\
=K_{m^{\prime}}\left(\cos \left(\frac{2 m^{\prime} \xi}{J^{\prime}(Y)}\right)=-1, Y\right)
\end{array}
$$

when $m^{\prime}$ is even. This implies that

$$
\begin{gathered}
\left(Y-Y_{s}\right)-\left(Y-Y_{s}\right)^{2}-\frac{\kappa}{2 m^{\prime} \sqrt{\delta}}\left(J(Y)-J\left(Y_{s}\right)\right. \\
\pm \frac{\epsilon}{2 \delta}\left(Y \mathcal{B}_{m^{\prime}}(Y)+Y_{s} \mathcal{B}_{m^{\prime}}\left(Y_{s}\right)\right)=0,
\end{gathered}
$$

where the + sign holds for odd $m^{\prime}$ and the - sign holds for even $m^{\prime}$. (Only the + case needs to be considered when $g<0$ and $\mu>0$.) 
Solutions $Y_{*}$ of (177) are perturbations to $Y_{m^{\prime}}$ of the form

$$
Y_{*}=Y_{m^{\prime}}+W \epsilon^{\gamma}+\mathcal{O}\left(\epsilon^{2 \gamma}\right)
$$

for an appropriate choice of $\gamma$, to be determined by a self-consistency argument. (When $\epsilon<0$, one writes (78) with $(-\epsilon)^{\gamma}$ instead. Everything stated here is otherwise the same in that situation.) In this analysis, one uses the fact that $Y_{s}=Y_{m^{\prime}} \pm \epsilon(\Delta Y)+\mathcal{O}\left(\epsilon^{2}\right)$, where the $+\operatorname{sign}$ is for odd $m^{\prime}$ and the $-\operatorname{sign}$ is for even $m^{\prime}$.

To find $W$ and $\gamma$, we insert $Y_{*}$ and $Y_{s}$ into (77) and expand the resulting expression in a power series about $\epsilon=0$. During this process, one obtains

$$
\begin{aligned}
Y_{*}^{2}= & Y_{m^{\prime}}^{2}+2 Y_{m^{\prime}} W \epsilon^{\gamma}+W^{2} \epsilon^{2 \gamma} \\
J\left(Y_{*}\right)= & J\left(Y_{m^{\prime}}\right)+\epsilon^{\gamma} W J^{\prime}\left(Y_{m^{\prime}}\right) \\
& \quad+\epsilon^{2 \gamma} W^{2} J^{\prime \prime}\left(Y_{m^{\prime}}\right)+\mathcal{O}\left(\epsilon^{3 \gamma}\right),
\end{aligned}
$$

which shows that that the only suitable value of $\gamma$ is $1 / 2$. Equating terms of order $\mathcal{O}(1)$ yields no new information. Equating terms of order $\mathcal{O}\left(\epsilon^{1 / 2}\right)$ yields the resonance relation (66). Equating terms of order $\mathcal{O}(\epsilon)$ shows that

$$
W=\left[ \pm \frac{Y_{m^{\prime}} \mathcal{B}_{m^{\prime}}\left(Y_{m^{\prime}}\right)}{\delta\left[1+\frac{\kappa}{2 m^{\prime} \sqrt{\delta}} J^{\prime \prime}\left(Y_{m^{\prime}}\right)\right]}\right]^{1 / 2}
$$

where the $+\operatorname{sign}$ occurs for odd $m^{\prime}$ and the $-\operatorname{sign}$ occurs for even $m^{\prime}$. Therefore, the miminal action of the resonance band is

$$
Y_{\text {min }}=Y_{m^{\prime}}-\sqrt{\epsilon} W+\mathcal{O}(\epsilon)
$$

and the maximal action is

$$
Y_{\max }=Y_{m^{\prime}}+\sqrt{\epsilon} W+\mathcal{O}(\epsilon) .
$$

The width of the resonance band is $Y_{\max }-Y_{\min }=$ $2 \sqrt{\epsilon} W+\mathcal{O}(\epsilon)$.

In Section [6] we compare these analytical results with numerical simulations.

\subsection{Attractive BECs with a Positive Chemical Potential}

Zounes and Rand ${ }^{53}$ considered (21) when $\delta>0$ and $\alpha>0$ (in other words, $\mu>0$ and $g<0$ ), which is depicted in Figure 1b. They did not consider the application of their analysis to Bose-Einstein condensates, so we presently interpret their results in this new light and compare it to our analysis of the repulsive case.
When $\delta>0, \alpha>0$, the phase space of the integrable problem contains no separatrix, and the entire space is foliated by periodic orbits. (See Table 11) This choice of parameters also leads to the simplest application of the perturbation technique described in Section 5.1. In this case, $k \in[0,1]$, so one need not apply a modulus transformation in the elliptic function solution. One may also set $\sigma=1$.

We refer the reader to Zounes and Rand ${ }^{53}$ for details. Here, we highlight a few results that we wish to contrast directly. When $g<0$ and $\mu>0$, the resonance relation one obtains is

$$
\frac{\kappa}{\sqrt{\delta}}=2 m^{\prime} \Omega_{a}\left(P_{m^{\prime}}\right)
$$

where the frequency $\Omega_{a}(P)$ has a similar form to that of $\Omega$ described above. In this situation, $\Omega_{a}(P) \geq 1$, so subharmonic periodic orbits are present when

$$
\frac{\kappa}{\sqrt{\delta}} \geq 2 m^{\prime}
$$

which is the reverse inequality as that derived in the repulsive case. Hence, there exist regimes in which only $2: 1$ resonances are present, only $2: 1$ and $4: 1$ resonances are present, etc. In terms of BEC parameters, the condition (84) describing the onset of $2 m^{\prime}: 1$ resonance bands takes the form

$$
\kappa \geq 2 m^{\prime} \sqrt{\frac{2 m \mu}{\hbar}} .
$$

Because the inequalities in (62) and (85) are oppositely directed, adjustments to $\kappa$ and $\mu$ have the opposite effect in these two cases.

Additionally, in this case there is no alternating of signs in the location of saddles and centers in resonance bands, as there is when $g>0$ and $\mu>0$. Because the attractive case with a positive chemical potential is simpler than the one we studied, Zounes and Rand ${ }^{53}$ were able to obtain better predictions describing the location of saddles and centers and the width of resonance bands from a perturbation analysis like that discussed in Section 5.1

\subsection{Attractive BECs with a Negative Chemical Potential}

The most difficult case to consider is that of attractive BECs with a negative chemical potential. In (21), $\alpha>0$ and $\delta<0$ (i.e., $\mu<0$ ), so the integrable dynamics exhibit two homoclinic orbits. (See Figure 1f.) The perturbative approach used in this paper must be applied separately inside and outside 
the separatrix. Orbits inside the separatrix satisfy $h<0$, those on the separatrix satisfy $h=0$, and those outside the separatrix satisfy $h>0$.

Inside the separatrix, $k \in(1, \infty)$, so one must apply the reciprocal modulus transformation to the arguments of the elliptic functions (23), (24). The sign of $\sigma$ determines whether one is considering perturbations of periodic orbits in the right half or left half of the phase plane. To utilize our perturbative analysis outside the separatrix, one must expand elliptic functions and elliptic integrals in power series about infinity, where $k=0$. This requires delicate numerical computations of Laurent series coefficients.

In principle, one can overcome the increased technical challenges present in this third case (which is also of interest) and apply the same analysis as in Section 5.1 but the lengthy calculations involved would entail a separate publication.

\section{Numerical Simulations}

To compare the analytical results in Section 5 with numerical simulations, we utilize $(R, S)$ coordinates with $m=1 / 2$ and $\hbar=1$. To lowest order in $\epsilon$, the change of variables $Y \longrightarrow P \longrightarrow j$ is a near-identity transformation, so $Y=j+\mathcal{O}(\epsilon)$. Recall from (32) that

$$
\begin{aligned}
j & =\frac{1}{2} \rho^{2}, \\
H_{0} & =h(j)=j-j^{2}=\frac{1}{2} s^{2}+\frac{1}{2} r^{2}-\frac{1}{4} r^{4},
\end{aligned}
$$

where $s=\partial r / \partial \chi$. For this comparison, we let $\alpha^{\prime}=$ 1 and vary $\kappa, \delta \equiv \mu$, and $\epsilon \equiv-2 m V_{0} / \hbar^{2}$. Recall additionally from (29) that

$$
r=\sqrt{\frac{g}{\hbar \mu}} R=\sqrt{\frac{\alpha^{\prime}}{\delta}} R, \quad s=\frac{1}{\mu} \sqrt{\frac{g}{2 m}} R^{\prime}=\frac{\sqrt{\alpha^{\prime}}}{\delta} R^{\prime} .
$$

\subsection{Methodology}

Before discussing our results, we briefly overview our comparison procedure.

The "exact" locations of saddles and centers and sizes of resonance bands were determined using direct numerical simulations of Poincaré sections of (21). The surface of section we employed satisfies $x_{n}=$ $2 n \pi / \kappa(n \in \mathbb{Z})$, which consists of integer multiples of the periodicity of the sinusoidal forcing in (21). In our simulations, the variable $\kappa x$ is periodic, so the surface of section is defined by the condition $\kappa x=0$. We used this framework to find saddles, centers, and resonance band sizes (i.e., separatrix widths) empirically.

To obtain our predictions, we employed the resonance Hamiltonian (65), whose level curves correspond to invariant curves of Poincaré sections. As each trajectory yields a level set of this Hamiltonian, we solved $K_{m}^{\prime}=$ constant numerically at appropriate energy values to obtain predictions for the locations of saddles and centers and the size of resonance bands; these latter quantities are determined from the widths of separatrices in (65). For these computations, we expanded elliptic functions and elliptic integrals in Taylor series and subsequently transformed these results to $(R, S)$-space to compare these calculations with our empirical ones. We also predicted the locations of saddles and centers (72/73) and the size of resonances bands 80 8182 using the predictions obtained from further perturbation expansions. We again tranformed back to $(R, S)$ space to compare this second set of predictions with our empirical results.

\subsection{Primary Resonances}

Our comparison between theory and numerics for primary resonances is summarized in Tables 2 and 3.

Consider first $\kappa=1.5$ and $\delta=1$. Poincaré sections and level sets of the resonance Hamiltonian $K_{1}$ [in units of $\left.\xi / J^{\prime}(Y)\right]$ are depicted in Figure [5] The results of our comparison between perturbation theory and numerical simulations are summarized in Table 2

We do relatively well in locating saddles and extremely well in locating centers. This is especially significant in light of the fact that many canonical transformations were required to obtain our analytical predictions. Although the requisite calculations are complicated, we are rewarded by excellent qualitative agreement and good (and sometimes excellent) quantitative agreement. For $\epsilon=0.05$, the numerical resolution of the location of the saddles was problematic, so a direct comparison is necessarily less accurate. As a result, a range of values is sometimes indicated for the numerically determined location of saddles. Such difficulties with direct numerical simulation emphasize the importance of using qualitative analytical methods to study the features of resonance bands.

Our comparisons between perturbation theory and numerical simulations for $\kappa=0.75, \delta=0.2$ are summarized in Table 3

If desired, one can improve these quantitative predictions by including higher-order contributions in 
the perturbation expansions.

\subsection{Secondary Resonances}

Our comparison between theory and numerics for secondary resonances is summarized in Table 4

We study $4: 1$ resonances for $\kappa=2.5$ and $\delta=1$. No 2:1 resonances exist for this choice of parameters. The resonance Hamiltonian is depicted for $\epsilon=0.05$ in Figure [6] The corresponding Poincaré section is shown in Figure 7

When $\epsilon=0.01$, we observe numerically that centers are located at approximately $(R, S)=$ $( \pm 0.691, \pm 0.332)$. With $R= \pm 0.691$, we predict a value of $S= \pm 0.32384$. With $S= \pm 0.332$, we predict a value of $R= \pm 0.68362$. These predictions are remarkably good, as we have used leading-order perturbation theory to derive analytical predictions for $4: 1$ (secondary) resonances. However, they are not as good as those obtained for the location of saddles in this case or the location of centers for 2:1 (primary) resonances.

When $\epsilon=0.05$, numerical simulations suggest that centers are located at about $(R, S)=$ $( \pm 0.697, \pm 0.330)$. Using $R= \pm 0.697$ leads to a prediction of $S= \pm 0.31912$. Using $S= \pm 0.330$ leads to a prediction of $R= \pm 0.68721$.

\subsection{Tertiary Resonances}

Our comparison between theory and numerics for tertiary resonances is summarized in Table 5

We consider $6: 1$ resonances for $\kappa=3.8, \delta=1$, and $\epsilon=0.01$. No $2: 1$ resonances exist for this choice of parameters, but $4: 1$ resonances do exist. The resonance Hamiltonian is depicted for $\epsilon=0.01$ in Figure 8. The corresponding Poincaré section is shown in Figure 9 (The 4:1 resonance bands are not shown in this plot.)

The predictions for centers are not as good as those for saddles, but there is nevertheless good quantitative agreement between observation and prediction, especially considering that a leading-order perturbation method has been employed. Of course, given that higher-order resonances occupy smaller regions of phase space, the absolute errors indicate that these predictions are not as good as the same absolute errors would be when studying lower-order resonances. This caveat notwithstanding, our theoretical analysis does an excellent job of determining the location of resonances and offers a useful tool for locating highorder resonances (and thus studying band structure in great detail) in numerical simulations.

\section{Conclusions}

In this paper, we studied in depth the band structure of BECs in periodic lattices. We approached this problem using a coherent structure ansatz, in contrast to the Bloch wave ansatz of earlier studies. $^{26,39,52}$

Using a technically delicate perturbative approach relying on elliptic function solutions of the integrable NLS, we examined the spatial resonance structure (band structure) of coherent structure solutions of the NLS in considerable detail, providing both an analytical description and numerical verifications of this theory. We derived conditions for the onset of $2 m^{\prime}: 1$ spatial resonances for all integer $m^{\prime}$ and developed analytical expressions for the width of these resonance bands and the locations of saddles and centers therein. Comparison with numerical simulations of primary, secondary, and tertiary resonances illustrate the applicability of our analytical theory.

Utilizing a simpler perturbative approach that employs Lindstedt's method and multiple scale analysis, we also established wave number-amplitude relations for coherent structure solutions of the NLS with a periodic potential. In so doing, we explored $2: 1$ spatial resonances and illustrated the utility of phase space analysis for the study of band structure as well as the structure of modulated amplitude waves in BECs.

In sum, our perturbative approach does an excellent job of determining the location of resonances and analyzing their structure and offers a useful tool for locating high-order resonances (and thus studying BEC band structure in great detail) in numerical simulations. An important open direction, to be addressed in a future publication, is the extent to which the theory developed here is an effective starting point for studies of the chaotic dynamics of BECs.

\section{Acknowledgements}

Valuable conversations with Eric Braaten, Michael Chapman, Mark Edwards, Nicolas Garnier, Brian Kennedy, Yueheng Lan, Igor Mezić, Peter Mucha, and Dan Stamper-Kurn are gratefully acknowledged. We are especially grateful to Jared Bronski, Richard Rand, and Li You for several extensive discussions concerning this project and to Panos Kevrekidis, Boris Malomed, Alexandru Nicolin, and an anonymous referee for critically reading and offering useful 
suggestions that greatly improved this manuscript.

\section{References}

[1] Milton Abramowitz and Irene Stegun, editors. Handbook of Mathematical Functions with Formulas, Graphs, and Mathematical Tables. Number 55 in Applied Mathematics Series. National Bureau of Standards, Washington, D. C., 1964.

[2] G. L. Alfimov, P. G. Kevrekidis, V. V. Konotop, and M. Salerno. Wannier functions analysis of the nonlinear Schrodinger equation with a periodic potential. Physical Review E, 66(046608), October 2002.

[3] B. P. Anderson and M. A. Kasevich. Macroscopic quantum interference from atomic tunnel arrays. Science, 282(5394):1686-1689, November 1998 .

[4] M. H. Anderson, J. R. Ensher, M. R. Matthews, C. E. Wieman, and E. A. Cornell. Observation of Bose-Einstein condensation in a dilute atomic vapor. Science, 269(5221):198-201, July 1995.

[5] Neil W. Ashcroft and N. David Mermin. Solid State Physics. Brooks/Cole, Australia, 1976.

[6] B. B. Baizakov, V. V. Konotop, and M. Salerno. Regular spatial structures in arrays of BoseEinstein condensates induced by modulational instability. Journal of Physics B: Atomic Molecular and Optical Physics, 35:5105-5119, 2002.

[7] Y. B. Band, I. Towers, and Boris A. Malomed. Unified semiclassical approximation for BoseEinstein condensates: Application to a BEC in an optical potential. Physical Review $A$, 67(023602), February 2003.

[8] Kirstine Berg-Sørensen and Klaus Mølmer. Bose-Einstein condensates in spatially periodic potentials. Physical Review A, 58(2):1480-1484, August 1998.

[9] Jared C. Bronski, Lincoln D. Carr, Ricardo Carretero-González, Bernard Deconinck, J. Nathan Kutz, and Keith Promislow. Stability of attractive Bose-Einstein condensates in a periodic potential. Physical Review E, 64(056615), 2001.
[10] Jared C. Bronski, Lincoln D. Carr, Bernard Deconinck, and J. Nathan Kutz. Bose-Einstein condensates in standing waves: The cubic nonlinear Schrödinger equation with a periodic potential. Physical Review Letters, 86(8):1402-1405, February 2001.

[11] Jared C. Bronski, Lincoln D. Carr, Bernard Deconinck, J. Nathan Kutz, and Keith Promislow. Stability of repulsive Bose-Einstein condensates in a periodic potential. Physical Review E, 63(036612), 2001.

[12] Lutz Brusch, Alessandro Torcini, Martin van Hecke, Martin G. Zimmermann, and Markus Bär. Modulated amplitude waves and defect formation in the one-dimensional complex Ginzburg-Landau equation. Physica D, 160:127148, 2001.

[13] Lutz Brusch, Martin G. Zimmermann, Martin van Hecke, Markus Bär, and Alessandro Torcini. Modulated amplitude waves and the transition from phase to defect chaos. Physical Review Letters, 85(1):86-89, July 2000.

[14] S. Burger, F. S. Cataliotti, C. Fort, F. Minardi, and M. Inguscio. Superfluid and dissipative dynamics of a Bose-Einstein condensate in a periodic optical potential. Physical Review Letters, 86(20):4447-4450, May 2001.

[15] Keith Burnett, Mark Edwards, and Charles W. Clark. The theory of Bose-Einstein condensation of dilute gases. Physics Today, 52(12):37-42, December 1999.

[16] Lincoln D. Carr, Charles W. Clark, and Wilhelm P. Reinhardt. Stationary solutions of the one-dimensional nonlinear Schrödinger equation. i. Case of repulsive nonlinearity. Physical Review A, 62(063610):1-10, 2000.

[17] Lincoln D. Carr, Charles W. Clark, and Wilhelm P. Reinhardt. Stationary solutions of the one-dimensional nonlinear Schrödinger equation. ii. Case of attractive nonlinearity. Physical Review A, 62(063611):1-10, 2000.

[18] Ricardo Carretero-González and Keith Promislow. Localized breathing oscillations of BoseEinstein condensates in periodic traps. Physical Review A, 66(033610), September 2002. 
[19] F. S. Cataliotti, L. Fallani, F. Ferlaino, C. Fort, P. Maddaloni, and M. Inguscio. Superfluid current disruption in a chain of weakly coupled Bose-Einstein condensates. New Journal of Physics, 5:71.1-71.7, June 2003.

[20] Dae-Il Choi and Qian Niu. Bose-Einstein condensates in an optical lattice. Physical Review Letters, 82(10):2022-2025, March 1999.

[21] Vincent T. Coppola. Averaging of Strongly Nonlinear Oscillators Using Elliptic Functions. $\mathrm{PhD}$ thesis, Cornell University, August 1989.

[22] Vincent T. Coppola and Richard H. Rand. Chaos in a system with a periodically disappearing separatrix. Nonlinear Dynamics, 1:401-420, 1990 .

[23] Franco Dalfovo, Stefano Giorgini, Lev P. Pitaevskii, and Sandro Stringari. Theory of Bose-Einstein condensation on trapped gases. Reviews of Modern Physics, 71(3):463-512, April 1999.

[24] K. B. Davis, M.-O. Mewes, M. R. Andrews, N. J. van Druten, D. S. Durfee, D. M. Kurn, and W. Ketterle. Bose-Einstein condensation in a gas of sodium atoms. Physical Review Letters, 75(22):3969-3973, November 1995.

[25] Bernard Deconinck, B. A. Frigyik, and J. Nathan Kutz. Dynamics and stability of Bose-Einstein condensates: The nonlinear Schrödinger equation with periodic potential. Journal of Nonlinear Science, 12(3):169-205, 2002.

[26] Dimitri Diakonov, L. M. Jensen, C. J. Pethick, and H. Smith. Loop structure of the lowest Bloch band for a Bose-Einstein condensate. Physical Review A, 66(013604), 2002.

[27] Elizabeth A. Donley, Neil R. Claussen, Simon L. Cornish, Jacob L. Roberts, Eric A. Cornell, and Carl E. Weiman. Dynamics of collapsing and exploding Bose-Einstein condensates. Nature, 412:295-299, July 19th 2001.

[28] D. G. Fried, T. C. Killian, L. Willmann, D. Landhuis, S. C. Moss, D. Kleppner, and T.J. Greytak. Bose-Einstein condensation of atomic hydrogen. Physical Review Letters, 81:38113814, 1998.
[29] Herbert Goldstein. Classical Mechanics. Addison-Wesley Publishing Company, Reading, MA, 2nd edition, 1980.

[30] Markus Greiner, Olaf Mandel, Tilman Esslinger, Theodor Hänsch, and Immanuel Bloch. Quantum phase transition from a superfluid to a Mott insulator in a gas of ultracold atoms. Nature, 415, January 3, 2002.

[31] John Guckenheimer and Philip Holmes. Nonlinear Oscillations, Dynamical Systems, and Bifurcations of Vector Fields. Number 42 in Applied Mathematical Sciences. Springer-Verlag, New York, NY, 1983.

[32] E. W. Hagley, L. Deng, M. Kozuma, J. Wen, K. Helmerson, S. L. Rolston, and W. D. Phillips. A well-collimated quasi-continuous atom laser. Science, 283(5408):1706-1709, March 1999.

[33] Wolfgang Ketterle. Experimental studies of Bose-Einstein condensates. Physics Today, 52(12):30-35, December 1999.

[34] Thorsten Köhler. Three-body problem in a dilute Bose-Einstein condensate. Physical Review Letters, 89(21):210404, 2002.

[35] Derek F. Lawden. Elliptic Functions and Applications. Number 80 in Applied Mathematical Sciences. Springer-Verlag, New York, NY, 1989.

[36] Allan J. Lichtenberg and M. A. Lieberman. Regular and Chaotic Dynamics. Number 38 in Applied Mathematical Sciences. Springer-Verlag, New York, NY, 2nd edition, 1992.

[37] Pearl J. Y. Louis, Elena A. Ostrovskaya, Craig M. Savage, and Yuri S. Kivshar. Boseeinstein condensates in optical lattices: Bandgap structure and solitons. Physical Review A, 67(013602), 2003.

[38] M. Machholm, A. Nicolin, C. J. Pethick, and H. Smith. Spatial period-doubling in Bose-Einstein condensates in an optical lattice. Physical Review A, 69(043604), 2004. ArXiv:cond-mat/0307183

[39] M. Machholm, C. J. Pethick, and H. Smith. Band structure, elementary excitations, and stability of a Bose-Einstein condensate in a periodic potential. Physical Review A, 67(053613), 2003. 
[40] Boris A. Malomed, Z. H. Wang, P. L. Chu, and G. D. Peng. Multichannel switchable system for spatial solitons. Journal of the Optical Society of America B, 16(8):1197-1203, August 1999.

[41] C. Menotti, A. Smerzi, and A. Trombettoni. Superfluid dynamics of a Bose-Einstein condensate in a periodic potential. New Journal of Physics, 5(112):112.1-112.20, September 2003.

[42] Erich J. Mueller. Superfluidity and meanfield energy loops; hysteretic behavior in BoseEinstein condensates. Physical Review A, 66(063603), 2002.

[43] C. J. Pethick and H. Smith. Bose-Einstein Condensation in Dilute Gases. Cambridge University Press, Cambridge, United Kingdom, 2002.

[44] Mason A. Porter and Predrag Cvitanović. Modulated amplitude waves in Bose-Einstein condensates. Physical Review E, 69(047201), 2004. ArXiv: nlin.CD/0307032.

[45] Richard H. Rand. Topics in Nonlinear Dynamics with Computer Algebra, volume 1 of Computation in Education: Mathematics, Science and Engineering. Gordon and Breach Science Publishers, USA, 1994.

[46] Richard H. Rand. Lecture notes on nonlinear vibrations. a free online book available at http://www.tam.cornell.edu/randdocs/nlvibe45.pdf 2003.

[47] J. L. Roberts, N. R. Claussen, S. L. Cornish, E. A. Donley, E. A. Cornell, and C. E. Wieman. Observation of Bose-Einstein condensation in dilute atomic vapor. Physical Review Letters, 86:2001-2004, 2001.

[48] L. Salasnich, A. Parola, and L. Reatto. Periodic quantum tunnelling and parametric resonance with cigar-shaped Bose-Einstein condensates. Journal of Physics B: Atomic Molecular and Optical Physics, 35(14):3205-3216, July 2002.

[49] A. Trombettoni and A. Smerzi. Discrete solitons and breathers with dilute Bose-Einstein condensates. Physical Review Letters, 86(11):23532356, March 2001.

[50] E. T. Whittaker and G. N. Watson. A Course of Modern Analysis. Cambridge University Press, Cambridge, Great Britain, fourth edition, 1927.
[51] Stephen Wiggins. Introduction to Applied Nonlinear Dynamical Systems and Chaos. Number 2 in Texts in Applied Mathematics. SpringerVerlag, New York, NY, 1990.

[52] Biao Wu, Roberto B. Diener, and Qian Niu. Bloch waves and Bloch Bands of Bose-Einstein condensates in optical lattices. Physical Review A, 65(025601), 2002.

[53] Randolph S. Zounes and Richard H. Rand. Subharmonic resonance in the non-linear Mathieu equation. International Journal of Non-Linear Mechanics, 37:43-73, 2002.

[54] Daniel Zwillinger (Ed.). Standard Mathematical Tables and Formulae. CRC Press, Inc., Boca Raton, FL, 30th edition, 1996. 


\begin{tabular}{|c|c|c|c|}
\hline Equilibrium at $(0,0)$ & Equilibria at $\left(R_{*}, 0\right) \neq(0,0)$ & $g$ & $\mu$ \\
\hline Center & None & - & + \\
Center & None & 0 & + \\
Center & Saddles & + & + \\
Saddle & None & + & - \\
Saddle & None & 0 & - \\
Saddle & Centers & - & - \\
\hline
\end{tabular}

Table 1: Type of equilibria of (5) when $V(x)=0$, and $c=0$.

\begin{tabular}{|c|c|c|c|c|}
\hline \multicolumn{3}{|c|}{$\epsilon=0.01$} & \multicolumn{2}{|l|}{$\epsilon=0.05$} \\
\hline Quantity & Perturbative & Numerical & Perturbative & Numerical \\
\hline$Y_{1}$ & 0.28133 & $\bullet$ & 0.28133 & $\bullet$ \\
\hline$Y_{s}$ & 0.27949 & - & 0.27213 & - \\
\hline$R_{s}$ & \pm 0.7477 & \pm 0.66 & \pm 0.73775 & $\pm(0.56-0.66)$ \\
\hline$Y_{c}$ & 0.28317 & $\bullet$ & 0.29053 & $\bullet$ \\
\hline$R_{c}$ & \pm 0.7526 & \pm 0.757 & \pm 0.76227 & \pm 0.774 \\
\hline$Y_{\min }$ & 0.16175 & $\bullet$ & - & $\bullet$ \\
\hline$Y_{\max }$ & 0.40091 & - & - & - \\
\hline$R_{i n}$ & \pm 0.56877 & \pm 0.66 & - & - \\
\hline$R_{\text {out }}$ & \pm 0.89545 & \pm 0.85 & - & - \\
\hline$Y_{\min , 2}$ & 0.22203 & $\bullet$ & - & - \\
\hline$Y_{\max , 2}$ & 0.35807 & - & - & - \\
\hline$R_{i n, 2}$ & \pm 0.66638 & \pm 0.66 & - & - \\
\hline$R_{\text {out }, 2}$ & \pm 0.84626 & \pm 0.85 & - & - \\
\hline
\end{tabular}

Table 2: Comparison of perturbation theory and numerics for $2: 1$ resonances $(\kappa=1.5, \delta=1)$. In this Table, $Y_{1}$ is the action value of the primary resonance, $Y_{s}$ is the location of its nearby saddle, $\left(R_{s}, 0\right)$ is its location in $(R, S)$-coordinates, $Y_{c}$ is the location of the nearby center, $\left(R_{c}, 0\right)$ is its location in $(R, S)$-space, $Y_{\min }$ is the minimum action value of the separatrix determined from 81, $Y_{\max }$ is the maximum determined from (82), $R_{\text {in }}$ is where the inner separatrix crosses the $R$-axis, $R_{\text {out }}$ is where the outer separatrix crosses the $R$-axis, $Y_{\min , 2}$ and $Y_{\max , 2}$ are the minimum and maximum actions obtained by solving (77) numerically, and $R_{i n, 2}$ and $R_{o u t, 2}$ are their corresponding predictions of where the inner and outer separatrices cross the $R$-axis. The symbol $\bullet$ means a calculation is not applicable and - means it was not computed. 


\begin{tabular}{|c|c|c|c|c|}
\hline \multicolumn{2}{|r|}{$\epsilon=0.01$} & \multicolumn{3}{|c|}{$\epsilon=0.05$} \\
\hline Quantity & Perturbative & Numerical & Perturbative & Numerical \\
\hline$Y_{1}$ & 0.19443 & $\bullet$ & 0.19443 & $\bullet$ \\
\hline$Y_{s}$ & 0.18499 & - & 0.14718 & - \\
\hline$R_{s}$ & \pm 27202 & $\pm(0.19-0.20)$ & \pm 0.24264 & - \\
\hline$Y_{c}$ & 0.20389 & $\bullet$ & 0.24169 & - \\
\hline$R_{c}$ & \pm 0.28558 & \pm 0.2908 & \pm 0.31093 & \pm 0.335 \\
\hline$Y_{\min }$ & 0.07485 & $\bullet$ & - & $\bullet$ \\
\hline$Y_{\max }$ & 0.31402 & - & - & - \\
\hline$R_{\text {in }}$ & \pm 0.17303 & \pm 0.19 & - & — \\
\hline$R_{\text {out }}$ & \pm 0.35441 & \pm 0.37 & - & - \\
\hline$Y_{\min , 2}$ & 0.09644 & $\bullet$ & 一 & - \\
\hline$Y_{\max , 2}$ & 0.34904 & - & - & - \\
\hline$R_{i n, 2}$ & \pm 0.19640 & \pm 0.19 & - & - \\
\hline$R_{\text {out }, 2}$ & \pm 0.37366 & \pm 0.37 & - & - \\
\hline
\end{tabular}

Table 3: Comparison of perturbation theory and numerics for $2: 1$ resonances $(\kappa=0.75, \delta=0.2)$. The quantities computed are defined in the caption of Table 2

\begin{tabular}{|c||c|c||c|r|}
\multicolumn{7}{c|}{$\epsilon=0.01$} & Perturbative & Numerical \\
\hline Quantity & Perturbative & Numerical & 0.37358 & $\bullet$ \\
$Y_{2}$ & 0.37358 & $\bullet$ & 0.37036 & \pm \\
$Y_{s}$ & 0.37294 & $\bullet$ & \pm 0.86065 & \pm 0.88 \\
$R_{s}$ & \pm 0.86364 & \pm 0.88 & \pm 0.68293 & $\bullet$ \\
$S_{s}$ & \pm 0.68389 & \pm 0.687 & 0.37680 & $\bullet$ \\
$Y_{c}$ & 0.37422 & $\bullet$ & See text & $( \pm 0.697, \pm 0.330)$ \\
$\left(R_{c}, S_{c}\right)$ & See text & $( \pm 0.691, \pm 0.332)$ & 0.31670 & $\bullet$ \\
$Y_{\min }$ & 0.34814 & $\bullet$ & 0.43046 & $\bullet$ \\
$Y_{\max }$ & 0.39902 & $\bullet$ & 0.32989 & $\bullet$ \\
$Y_{\min , 2}$ & 0.35571 & $\bullet$ & 0.46240 & $\bullet$ \\
$Y_{\max , 2}$ & 0.41237 & $\bullet$ & & \\
\hline
\end{tabular}

Table 4: Comparison of perturbation theory and numerics for $4: 1$ resonances $(\kappa=2.5, \delta=1)$. Tha action value of the secondary resonance is denoted $Y_{2}$. Saddles that intersect the $R$-axis are denoted $\left(R_{s}, 0\right)$, and those that intersect the $S$-axis are denoted $\left(0, S_{s}\right)$. Centers are denoted $\left(R_{c}, S_{c}\right)$. The other quantities computed are defined in the caption of Table 2

\begin{tabular}{|c||c|r|}
\hline Quantity & Perturbative & Numerical \\
\hline$Y_{3}$ & 0.36857 & $\bullet$ \\
$Y_{s}$ & 0.36851 & $\bullet$ \\
$R_{s}$ & \pm 0.85850 & $\pm(0.859-0.860)$ \\
$Y_{c}$ & 0.36863 & $\bullet$ \\
$R_{c}$ & \pm 0.85864 & \pm 0.870 \\
$Y_{\min }$ & 0.36214 & $\bullet$ \\
$Y_{\max }$ & 0.37500 & $\bullet$ \\
$Y_{\min , 2}$ & 0.36614 & $\bullet$ \\
$Y_{\max , 2}$ & 0.38653 & $\bullet$ \\
\hline
\end{tabular}

Table 5: Comparison of perturbation theory and numerics for $6: 1$ resonances $(\kappa=3.8, \delta=1, \epsilon=0.01)$. Tha action value of the tertiary resonance is denoted $Y_{3}$. The other quantities computed are defined in the caption of Table 2 


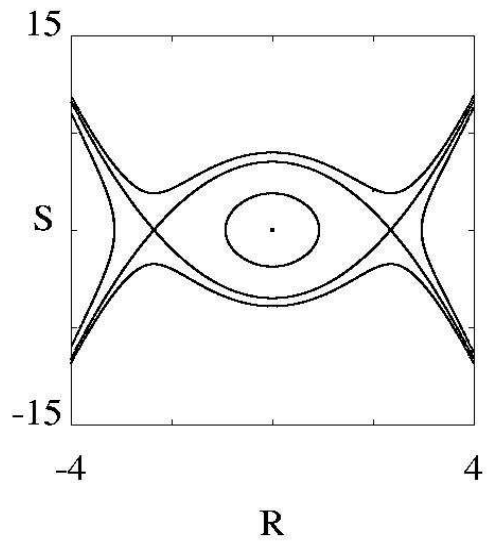

(a)

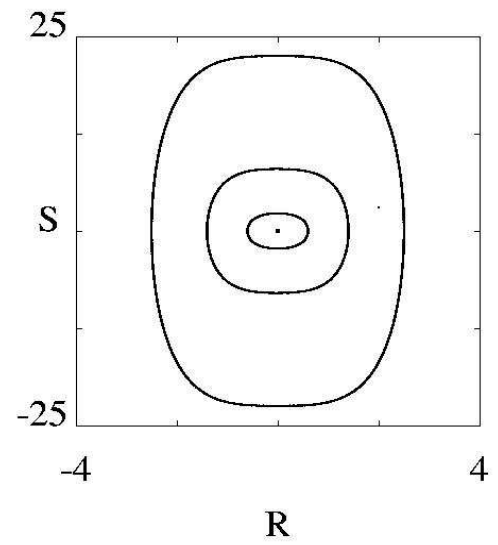

(b)

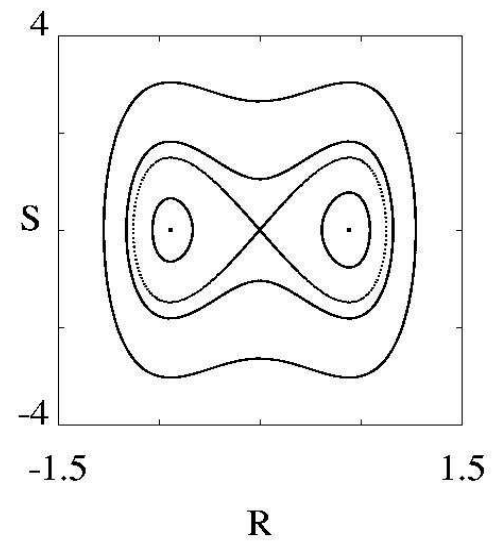

(c)

Figure 1: Phase portraits of coherent structures in BECs with no external potential. The signs of $\mu$ and $g$ determine the dynamics of (5). (a) Repulsive BEC with $\mu>0$. The two-body scattering length is $a=0.072$ $\mathrm{nm}$, the value ${ }^{28}$ for atomic hydrogen $\left({ }^{1} \mathrm{H}\right)$. Orbits inside the separatrix (which consists of two heteroclinic orbits) have bounded amplitude $R(x)$. The period of such orbits increases as one approaches the separatrix, whose period is infinite. (b) Attractive BEC with $\mu>0$. The two-body scattering length is $a=-0.9 \mathrm{~nm}$, the value ${ }^{25,47}$ for ${ }^{85} R b$. (c) Attractive BEC (again ${ }^{85} R b$ ) with $\mu<0$. Here there are two separatrices, each of which encloses periodic orbits satisfying $R \neq 0$. 


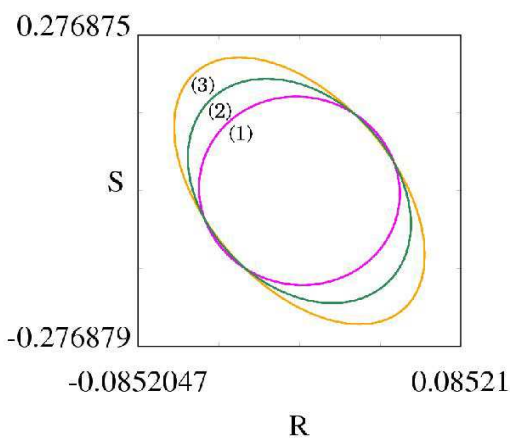

(a)

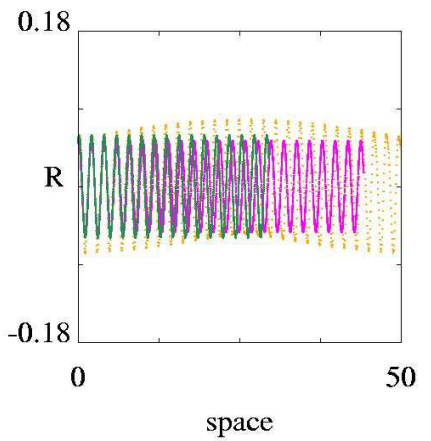

(c)

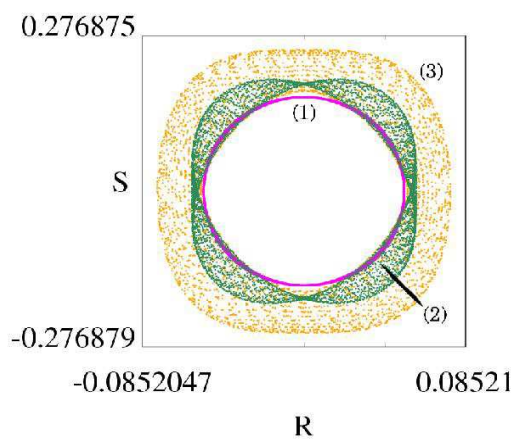

(b)

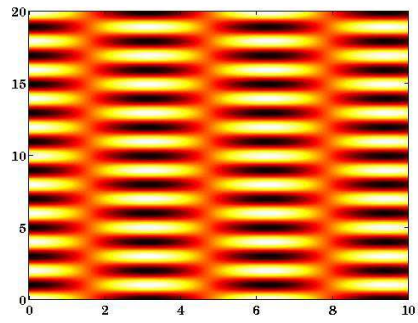

(d)

Figure 2: As the wave number $\kappa$ of the perturbation is increased, periodic behavior persists for larger $\left|V_{0}\right|$. The initial condition in this plot is $(R(0), S(0))=(0.05,0.05)$, and the parameter values $a=0.072$, $\mu=10, m=0.5$, and $x_{0}=0$ are used for each trajectory. (a) Poincaré section determined by $\sin (\kappa x)=0$. Trajectory $(1)$ corresponds to $\left(\kappa, V_{0}\right)=(100,10)$, trajectory $(2)$ to $\left(\kappa, V_{0}\right)=(100,100)$, and trajectory $(3)$ to $\left(\kappa, V_{0}\right)=(10,10)$. These quasiperiodic solutions indicate the existence of nearby periodic orbits. (b) Phase space plots of the trajectories in (a). Trajectory (1) is the closest to being periodic and trajectory (3) is the furthest away. (c) Amplitude $R$ as a function of space $x$ for trajectories (1)-(3). The band structure of BECs can be studied not only in real space but also in phase space by plotting Poincaré sections and trajectories, as indicated in (a) and (b). Examining the proximity of a trajectory to periodicity is most easily accomplished in phase space. (d) Coherent structure corresponding to quasiperiodic trajectory (1). This plot depicts $\operatorname{Re}(\psi)$. The horizontal axis represents time, and the vertical one represents space. The darkest portions are the most negative, and the lightest are the most positive. 


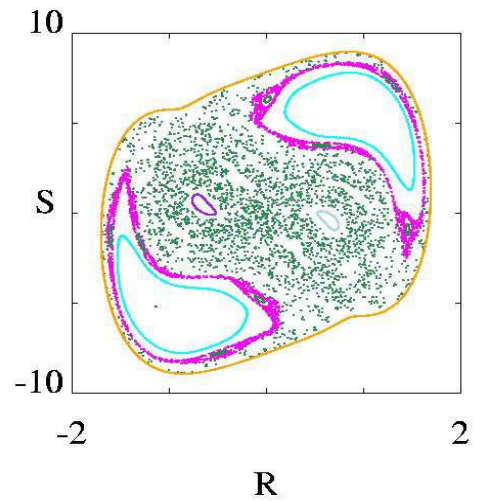

Figure 3: Poincaré section for the parameter values $\mu=-10, m=0.5, x_{0}=0, V_{0}=5, \kappa=10$, and $a=-0.9 \mathrm{~nm}$, corresponding to the experimentally determined scattering length ${ }^{25,47}$ for ${ }^{85} \mathrm{Rb}$. The depicted trajectories include examples which are quasiperiodic, locally chaotic (near the resonances), and globally chaotic (the stochastic sea).

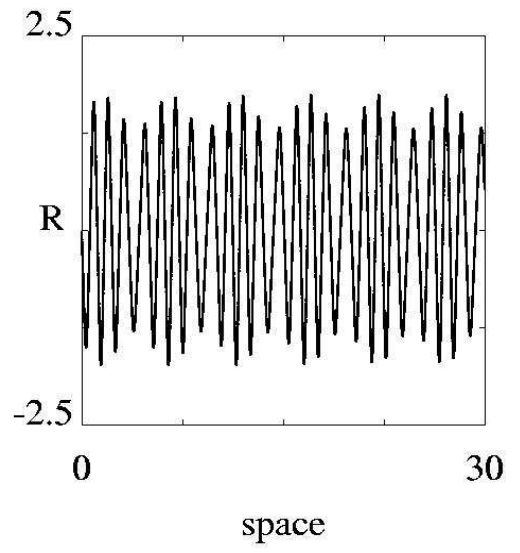

(a)

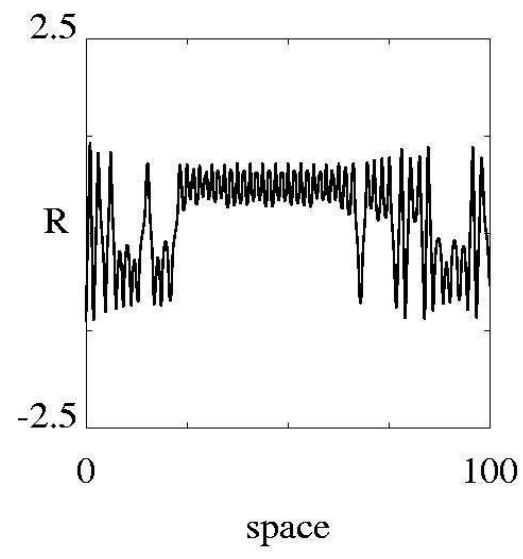

(b)

Figure 4: (a) Spatial profile of the coherent structure corresponding to the locally chaotic trajectory in Figure 3 The initial conditions are $(R(0), S(0)) \approx(-0.01818215,-5.23268358)$. (b) Spatial profile of the coherent structure corresponding to the globally chaotic trajectory in Figure 3 The initial conditions are $(R(0), S(0)) \approx(-1.13283530,1.28334013)$. 


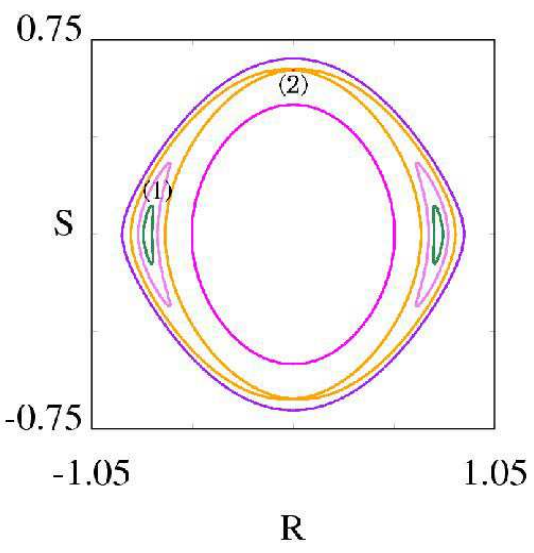

(a)

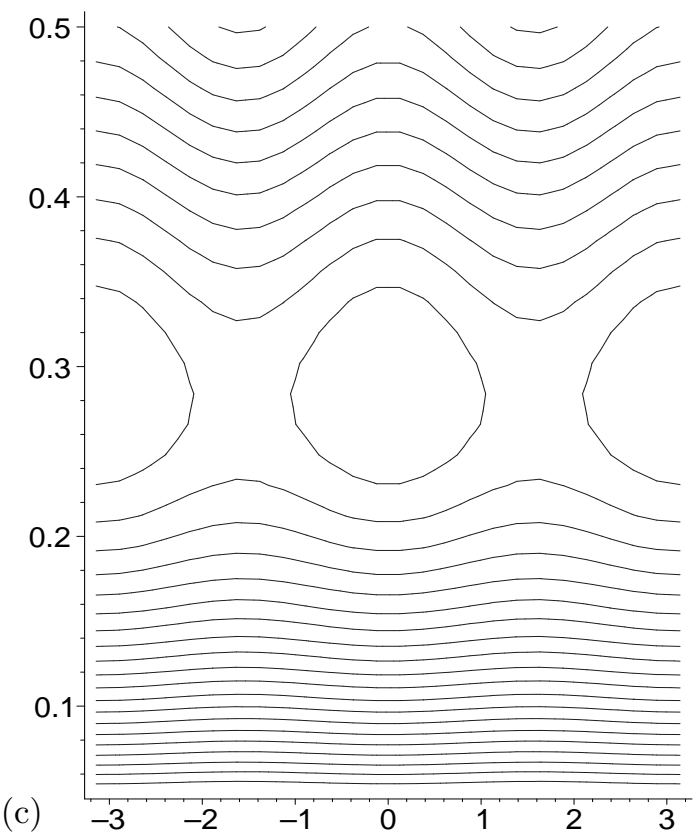

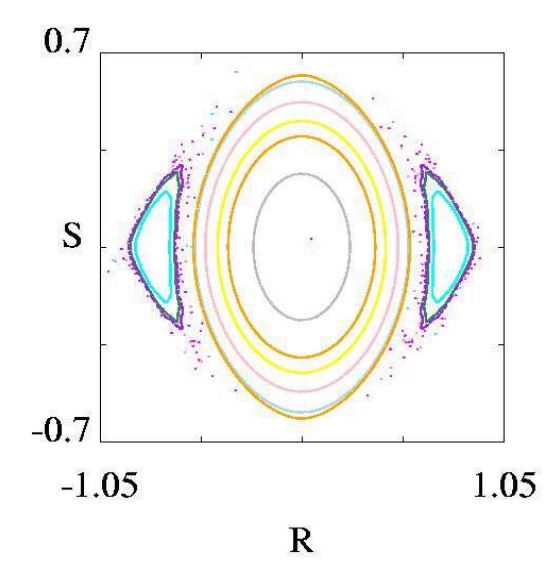

(b) 


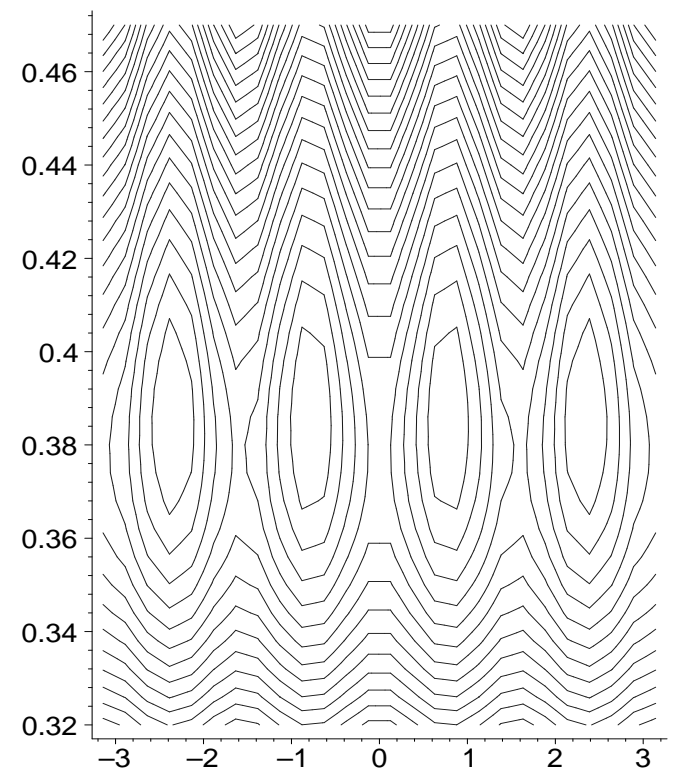

Figure 6: Resonance Hamiltonian $K_{2}$ for $\kappa=2.5, \delta=1$, and $\epsilon=0.05$.

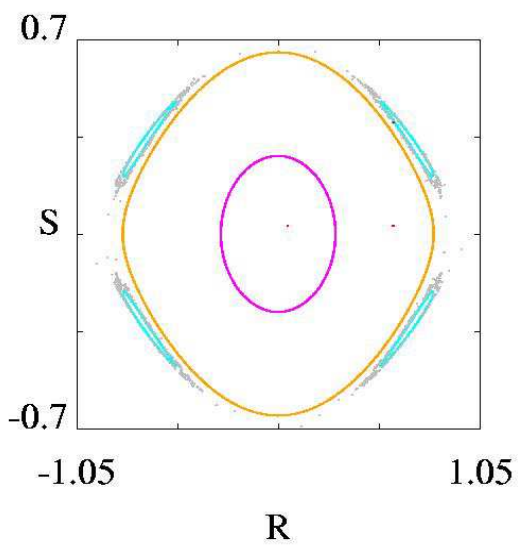

(a)

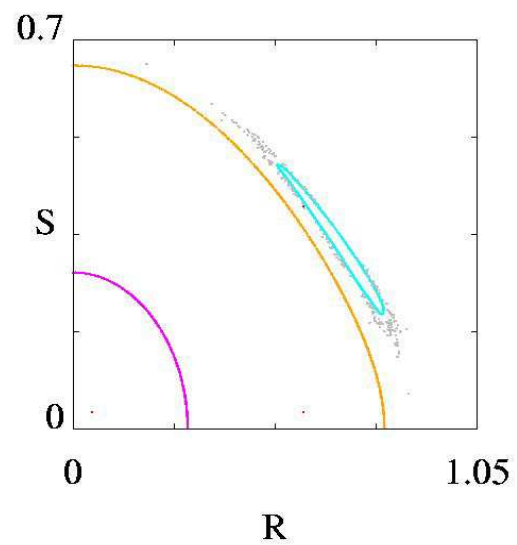

(b)

Figure 7: (a) Poincaré section for $\kappa=2.5, \delta=1$, and $\epsilon=0.05$. Note that there is no 2:1 resonance band for this choice of $(\kappa, \delta)$. The $4: 1$ resonance is depicted. (b) Upper right corner of (a). 


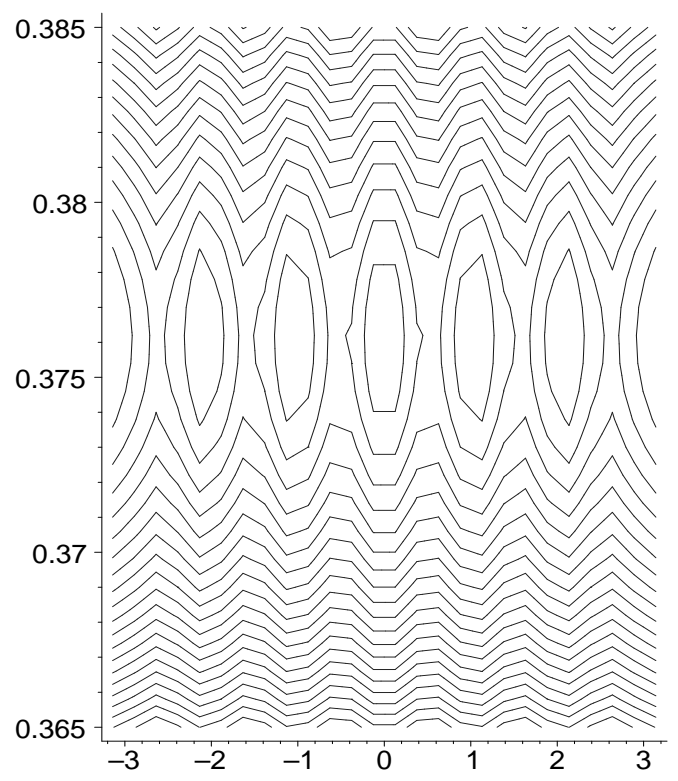

Figure 8: Resonance Hamiltonian $K_{3}$ for $\kappa=3.8, \delta=1$, and $\epsilon=0.01$.

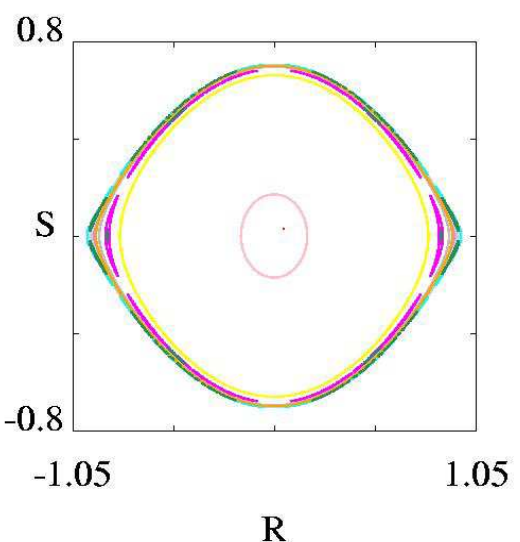

(a)

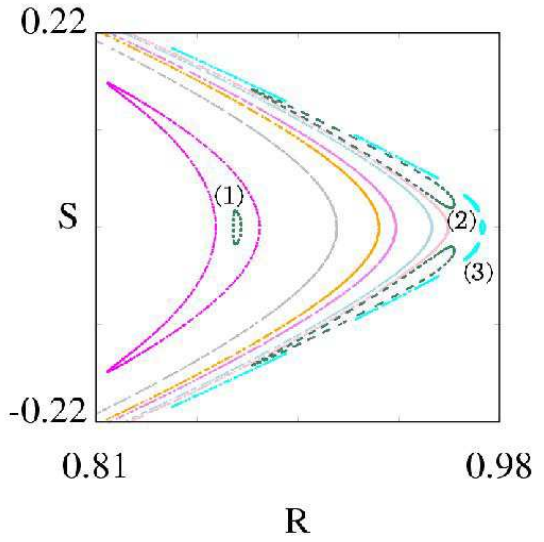

(b)

Figure 9: (a) Poincaré section for $\kappa=3.8, \delta=1$, and $\epsilon=0.01$. (b) Close-up of the resonances in (a). Both $6: 1$ (1) and 8:1 (2) resonances are displayed. A higher-order resonance (3) is also depicted. Although not shown, $4: 1$ resonances are also present for this choice of parameter values. 